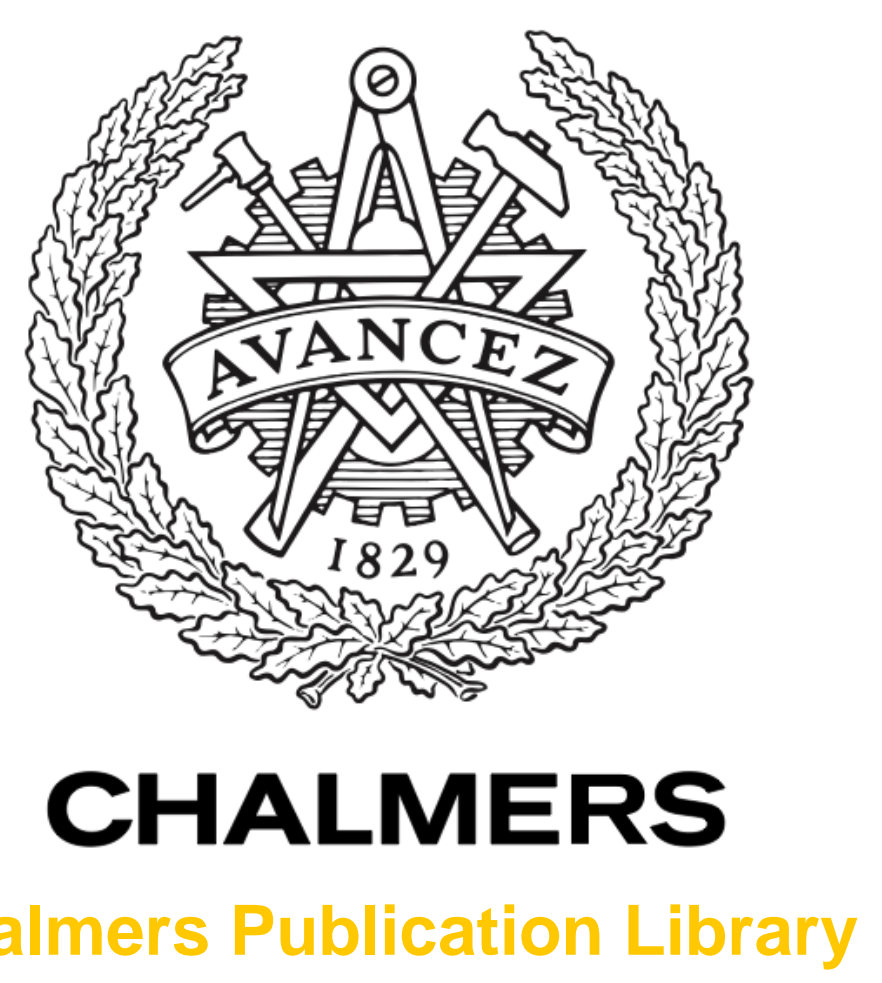

Chalmers Publication Library

\title{
A Review of Near-Collision Driver Behavior Models
}

This document has been downloaded from Chalmers Publication Library (CPL). It is the author's version of a work that was accepted for publication in:

Human Factors (ISSN: 0018-7208)

Citation for the published paper:

Markkula, G. ; Benderius, O. ; Wolff, K. et al. (2012) "A Review of Near-Collision Driver Behavior Models". Human Factors, vol. 54(6), pp. 1117-1143.

http://dx.doi.org/10.1177/0018720812448474

Downloaded from: http://publications.lib.chalmers.se/publication/171559

Notice: Changes introduced as a result of publishing processes such as copy-editing and formatting may not be reflected in this document. For a definitive version of this work, please refer to the published source. Please note that access to the published version might require a subscription. 


\title{
A review of near-collision driver behavior models
}

\author{
Gustav Markkula \\ Volvo Technology Corporation \\ Göteborg, Sweden
}

\author{
Ola Benderius, Krister Wolff and Mattias Wahde \\ Department of Applied Mechanics \\ Chalmers University of Technology \\ Göteborg, Sweden
}

\begin{abstract}
Objective: This paper provides a review of recent models of driver behavior in onroad collision situations.

Background: In the efforts to improve traffic safety, computer simulation of accident situations holds promise as a valuable tool, both for academia and industry. However, in order to ensure the validity of simulations, models are needed that accurately capture near-crash driver behavior, as observed in real traffic or driving experiments.

Method: Scientific papers were identified by a systematic approach, including extensive database searches. Criteria for inclusion were defined and applied, including the requirement that models should have been previously applied to simulate on-road collision avoidance behavior. Several selected models were implemented and tested in selected scenarios.

Results: The reviewed papers were grouped according to a rough taxonomy based on main emphasis, namely: Avoidance by braking, avoidance by steering, avoidance by a combination of braking and steering, effects of driver states and characteristics on avoidance, and simulation platforms.

Conclusion: A large number of near-collision driver behavior models have been proposed. Validation using human driving data has often been limited, but exceptions exist. The research field appears fragmented, but simulation-based comparison indicates that there may be more similarity between models than what is apparent from the model equations. Further comparison of models is recommended.

Application: This review provides traffic safety researchers with an overview of the field of driver models for collision situations. Specifically, researchers aiming to develop simulations of on-road collision accident situations can use this review to find suitable starting points for their work.
\end{abstract}

Keywords: driver behavior, models, simulation, collisions, accidents, crashes, avoidance

Road traffic accidents are a global problem, causing enormous economic and social costs, and more than a million fatalities every year (World Health Organization, 2009). A considerable proportion of severe accidents involve on-road collisions (see e.g. Najm, Smith, \& Yanagisawa, 2007). It is widely accepted that the behavior of vehicle drivers contributes strongly to accident causation, and much research effort has therefore been directed at understanding the relationship between driver behavior and safety, as well as what can be done

This work was supported by a grant from the VINNOVA Swedish Governmental Agency for Innovation Systems (2009-02766). to avoid or improve behaviors associated with crashes (J. D. Lee, 2008).

A time-honored approach in these endeavors has been the description and prediction of human driver behavior by means of models (see e.g. Gibson \& Crooks, 1938). In recent years, some traffic safety researchers have applied quantitative driver behavior models in computer simulation. For example, simulation of road networks with many simulated drivers has been used to study the potential safety impact of envisioned infrastructure improvements (see, for example, Saka \& Glassco, 2001). Also, estimates of the expected reduction of accidents from driving support technology, such as collision warning systems, have been obtained from computer simulations of the final seconds leading up to a crash (see e.g. T. Brown, Lee, \& McGehee, 2001). 
In general, computer simulation can provide a means of obtaining data regarding a system under study in a manner that is more controlled, repeatable, cheap, fast, and safe than obtaining similar data from real-life measurements. As a consequence, simulations may allow more powerful statistical analyses, or more complete testing and comparison of large numbers of alternative system designs. Computer simulations therefore hold great promise as an important tool for traffic safety research and development, both within industry and academia.

However, there is one general constraint: The results of simulations will never be more valid than the models on which the simulations are based. In order to realize any of the above-mentioned benefits of computer simulation of accidents, a crucial requirement is the availability of well-defined, quantitative models that accurately capture the behavior of drivers in the considered accident situations.

A number of authors have reviewed the driver modeling literature, from various perspectives (Reid, 1983; Michon, 1985; Ranney, 1994; Ghazi Zadeh, Fahim, \& El-Gindy, 1997; Brackstone \& McDonald, 1999; Helbing, 2001; MacAdam, 2003; X. Wang, Yang, Shan, \& Wang, 2006; Cody \& Gordon, 2007; Plöchl \& Edelmann, 2007; Weir \& Chao, 2007; Jürgensohn, 2007; Oppenheim et al., 2010). Among these, only Reid (1983) focused specifically on the modeling of accident-related driving behavior, concluding that at the time of writing there was "no well-developed and validated model for the detailed study of accidents" (p. 23). The other listed reviews all addressed models of driving behavior in general, with no specific focus on accident situations, and many also focused on models that were qualitative rather than quantitative, or models that for other reasons were not specified to the extent needed for implementation in computer simulation.

The aim of this review is to describe recent simulation-ready models of driver behavior in accident situations involving on-road collisions. The limitation to on-road collision accidents is adopted to keep the review manageable in size.

The remainder of the text will be organized as follows: First, some background will be provided on current theory and empirics regarding on-road collision accidents. Then, the method for identifying suitable models to review will be presented, including inclusion and exclusion criteria. Next, the identified models will be presented. In a concluding section, the models will be discussed and compared to the theoretical and empirical accounts of accident causation. Some suggestions for future work will be given as well.

\section{Background}

A brief presentation regarding theoretical and empirical results on driver behavior in near-collision situations will now be given, in order to provide a general outline of the reality that the driver models reviewed in this paper typically should aim to reproduce.

Based on U.S. accident statistics, Najm et al. (2007) proposed a typology of 37 pre-crash scenarios. A majority of these involved on-road collisions, with motor vehicles, cyclists, pedestrians, animals or other objects, occurring both at intersections and non-intersection locations. Examples of collision accident classes that can be found in the paper are: (a) rear-end collisions, with sub-classes lead vehicle decelerating (LVD), lead vehicle moving (LVM), and lead vehicle stationary (LVS); (b) intersection collisions; and (c) head-on collisions.

As a support when reasoning about the transition from normal driving into accidents, many authors have introduced divisions of the pre-crash timeline into a sequence of states. The division adopted by Najm and Smith (2004) is shown in the left part of Figure 1.

\section{Non-critical collision avoidance}

In everyday driving, a driver will routinely pass from a low risk state into the conflict state, for example whenever a slower moving lead vehicle is encountered, and then back again to the low risk state as a result of successful use of acceleration, deceleration, steering, or a combination thereof.

Several accounts have been proposed regarding how such everyday collision avoidance is achieved. A recurring concept in these is satisficing: Drivers will normally not apply collision avoidance at the very instant a collision course is established (which could be referred to as optimizing), but instead at some later time, related to the safety margins of the driver (see e.g. Summala, 2007).

D. Lee (1976) introduced the quantity $\tau=\theta / \dot{\theta}$, where $\theta$ is the angle subtended by an obstacle on the driver's retina, and demonstrated that $\tau$ is a close estimate of time to collision (TTC). Furthermore, he proposed that drivers initiate braking when $\tau$ passes a certain threshold, independent of speed, and he also demonstrated how $\dot{\tau}$, the time derivative of the same quantity, could hypothetically be used during control of braking. Fajen $(2005,2008)$ proposed another model, integrating satisficing aspects also in the control of an ongoing braking 


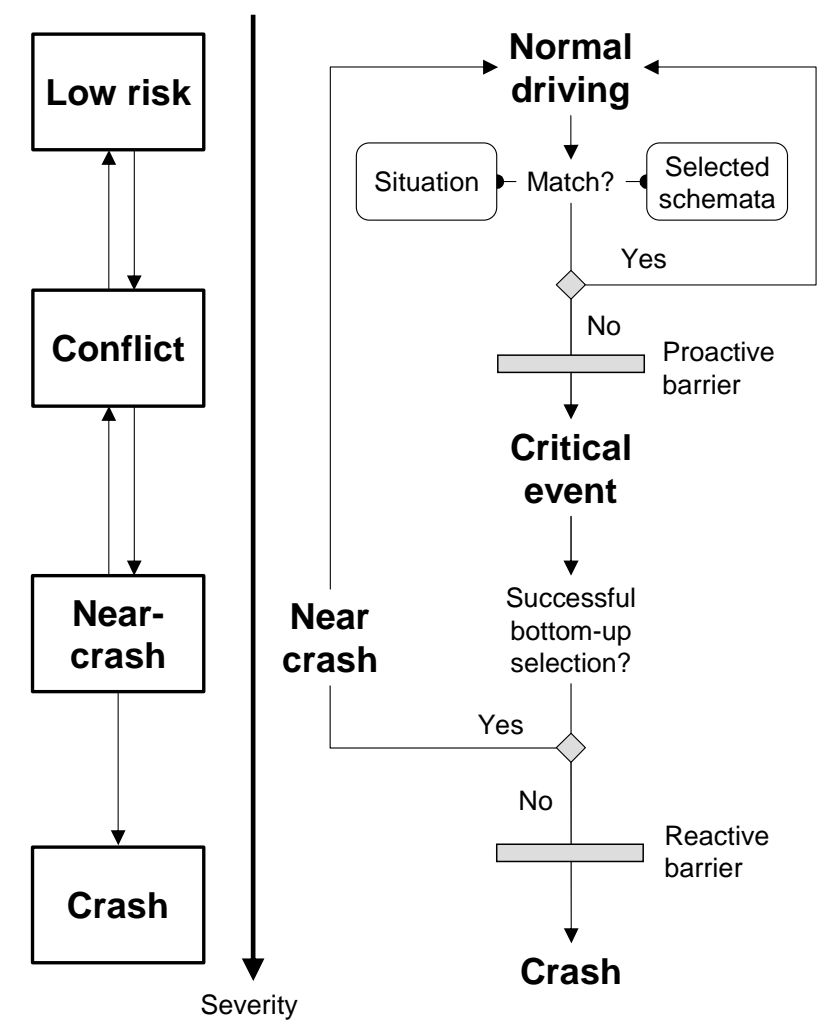

Figure 1. : The four driving conflict states adopted by Najm and Smith (2004), alongside the crash causation model of Engström et al. (in press).

maneuver. Kiefer, LeBlanc, and Flannagan (2005) analyzed a large dataset of test track driving, and found that test subject brake initiation could be described as occurring at a speed-dependent threshold for inverse TTC, decreasing linearly with increasing speed.

Timing of steering initiation in normal avoidance may be subject to the same general patterns, although with later timing than braking responses (Kiefer et al., 2005; Najm \& Smith, 2004). As for braking, models are available regarding the visual cues that are used by drivers during steering, and how these cues are translated into control actions (Land \& Horwood, 1995; Wann \& Wilkie, 2004).

It has been repeatedly observed that drivers are capable of adapting their control behavior to the specific vehicle they are driving (MacAdam, 2003). Nevertheless, experiments on open-loop control (where drivers are deprived of visual and inertial feedback) also sug- gest that drivers' understanding of the dynamics of their vehicles may be fundamentally limited (Cloete \& Wallis, 2009).

\section{Transitions to critical collision events}

Sometimes, normal driving passes into more critical states. A near-crash state can be defined, for example, in terms of the kinematics of the momentary traffic situation (Najm \& Smith, 2004), or in terms of the severity of required avoidance maneuvering (S. E. Lee, Llaneras, Klauer, \& Sudweeks, 2007). The crash state can be regarded as being reached when it is no longer possible to avoid the collision.

It is still a matter of scientific debate exactly why these transitions from normal to critical driving sometimes occur. Accident statistics and empirical studies point to many factors that correlate with accident risk, such as fatigue, alcohol intoxication, distractions, age, driving experience, and driving style (see e.g. J. D. Lee, 2008 for an overview), and a variety of competing qualitative models have been proposed for explaining how these factors come into play.

One view is available from information processing models of human behavior. In this paradigm, human behavior in general has been described as the result of information processing along a sequence of stages, for example: (a) sensory processing, (b) perception, (c) cognition and memory, (d) response selection, and (e) response execution (Wickens \& Hollands, 2000). Accidents have been modeled as due to errors, occurring at different points along the sequence of processing, causing degradation of the normal behavior in the form of, for example, slips, lapses, or mistakes (Wickens \& Hollands, 2000). The frequency of such errors can then be assumed to vary with accident-related factors such as those listed above. van Elslande and Fouquet (2007) provided one example of how a model based on information processing can be applied in the study of traffic accident causation.

Alternatives to this type of model exist and one example, focusing mainly on the role of attention in accident causation, is illustrated alongside the pre-crash timeline division in Figure 1. This model is due to Engström, Victor, and Markkula (in press; see also Engström, 2011), who discussed driver behavior using the metaphor of schemata, "functional units of action control at different levels of abstraction" (p. 38), such as for example recognize traffic light, or follow the car ahead. They hypothesized that, under normal circumstances, proper schemata selection (based on the driver's understanding of how the traffic situation will 
evolve in the near future) is sufficient to avoid critical events. Engström et al. referred to this mechanism as a proactive barrier, and proposed that it fails when there is a mismatch between the selected schemata and the traffic situation at hand, such that early conflict resolution is either unsuccessful or absent altogether. Candidate factors which could be hypothesized to increase the risk of such mismatches include infrequent events, misleading contextual cues, and cognitive distractions. For example, cognitive distraction has been observed to impair driver ability to adapt gap acceptance judgments to road conditions, when turning at an intersection (Cooper \& Zheng, 2002), and in straight-line collision situations, unexpected braking stimuli and cognitive distractions have been empirically linked with later brake reactions (Green, 2000; Salvucci, 2002).

In the model proposed by Engström et al., when the proactive barrier fails, the crash may still be prevented by the reactive barrier: Visual stimuli, such as the looming of the obstacle on the retina, are hypothesized to cause bottom-up reflex activation of near-crash avoidance schemata. Recent naturalistic driving studies have highlighted the strong relationship between visual distraction and crashes (see e.g. Dingus et al., 2006; S. E. Lee et al., 2007), and Engström et al. proposed that these observations could be understood in terms of the reactive barrier failing when the driver's gaze is off the road ahead. It has been shown that the most common driver behavior in rear-end crashes is no maneuver at all (S. E. Lee et al., 2007; Wiacek \& Najm, 1999), and in accident statistics this was linked to driver distraction by Yan, Harb, and Radwan (2008).

\section{Critical collision avoidance}

Models of why a near-crash collision situation may arise, such as outlined above, can be complemented with models of more exactly when and how near-crash collision avoidance maneuvering is then carried out by the driver.

Maneuver timing has been studied by many researchers, in terms of the reaction time from a stimulus to an evasive reaction, and reaction time estimates have been proposed as functions of a range of parameters: Stimulus eccentricity, number of obstacles, nighttime versus daytime driving, age, gender, as well as the above-mentioned cognitive distraction and stimulus expectancy (Delaigue \& Eskandarian, 2004; Green, 2000; Muttart, 2003; B. Wang, Abe, \& Kano, 2002). In addition, limitations on just noticeable differences of changing stimuli, as prescribed by Weber's law (Gray, 2010, p. 263), have also been discussed as introducing con- straints regarding the earliest time at which a driver can react to a change in the traffic scene (see e.g. D. Lee, 1976).

As for the manner in which drivers carry out critical collision avoidance maneuvering, braking only (without steering) has been identified as the most natural first response for most drivers, and steering (alone or with braking) has been observed more frequently at low values of TTC, such that the driver may perceive that braking is insufficient to avoid the crash (Adams, 1994; S. E. Lee et al., 2007; Wiacek \& Najm, 1999) ${ }^{1}$.

Several researchers have noted a tendency of drivers not to apply steering to the full stability limits of their vehicles, and to brake and collide in situations where steering could have avoided the collision. It has been proposed that such behavior may be due to (a) drivers having very little experience of applying high lateral accelerations, or (b) perceived added risks from abruptly steering away from one's own lane (Adams, 1994; Lechner \& van Elslande, 1997). Breuer (1998) compared normal driving in real traffic with driving in a double lane change maneuver (similar to that of ISO, 1999), and argued that the high lateral accelerations induced in such test track maneuvers are very rare in real traffic.

Braking at the vehicle's limits seems more common (Lechner \& van Elslande, 1997; McGehee et al., 1999), but also in this context it has been argued that lack of experience of, and low expectancy for, critical braking may limit the magnitude of avoidance maneuvering employed by drivers (Dilich, Kopernik, \& Goebelbecker, 2002).

In general, an important question is whether the models of non-critical collision avoidance presented earlier are valid in more critical situations. Hollnagel and Woods (2005, p. 146) defined different control modes and argued that, whereas control often relies on anticipation and planning (tactical and strategical control modes), in more urgent or unusual situations control may rather be driven by salient features of the immediate situation (opportunistic control mode) or even become random (scrambled control mode). This relates clearly to the above-mentioned observations of nonresponse behavior in crashes (S. E. Lee et al., 2007; Wiacek \& Najm, 1999), and also to reports from accident reconstructions on driver control overreactions (Malaterre, Ferrandez, Fleury, \& Lechner, 1988).

${ }^{1}$ S. E. Lee et al. (2007) also observed steering responses in situations with high TTC, where there was presumably enough time for the driver to plan a more controlled maneuver, but this relates less to critical collision avoidance. 


\section{Collision avoidance support systems}

Finally, an important aspect of driver behavior in relation to collision accidents is driver response to warnings and interventions from in-vehicle support systems, which may hold potential for helping drivers avoid or mitigate collision accidents, for example by redirecting gaze of visually distracted drivers to the collision threat (see, for instance, J. D. Lee, McGehee, Brown, $\&$ Reyes, 2002), or by applying autonomous braking or steering of the vehicle (Itoh, Horikome, \& Inagaki, 2010). A complication in this context is the phenomenon of behavioral adaptation, by which driver response to a support system may change over time with exposure to the system (Smiley, 2000). The driver's long-term behavior with respect to a support system, in terms of acceptance, reliance, disuse, or even misuse of the system, may depend on a multitude of factors. Examples include perceived system reliability, the degree to which the system can be understood by the driver, and the specifics of warning or intervention design (J. D. Lee \& See, 2004; Meyer, 2004).

\section{Method}

Candidate scientific papers for this review were gathered using a systematic approach, from the following sources: (a) Database searches (see Appendix A for details), (b) reference lists of candidate articles, (c) researcher web pages, and (d) previous knowledge of the authors and their colleagues. Both peer-reviewed and non-peer-reviewed publications were considered.

Inclusion of candidate papers in this review was then decided based on the following criteria: (a) They should be written in English, and published in the year 2000 or later; however, some influential references of older date were also included; (b) they should describe driver behavior models capable of controlling a simulated vehicle laterally and/or longitudinally, using some input from a simulated traffic situation, thus excluding papers describing models of, for example, perception only or actuation only; (c) they should address traffic on public roads, as opposed to race track driving; and (d) they should describe simulation of near-crash on-road collision situations. No strict definition of what constitutes a near-crash situation (in terms of, for example, kinematics or response severity, as discussed above) was adopted, since candidate articles would rarely give information regarding such details. Therefore, application of the final inclusion criterion involved subjective judgment to some degree. In borderline cases, we have opted for inclusion rather than exclusion. However, based on this criterion, papers reporting on the use of crash-free traffic simulation in combination with surrogate safety measures (see e.g. Saka \& Glassco, 2001) were consistently excluded.

Some of the driver models presented in the reviewed papers were implemented and simulated in selected scenarios; details will be provided as part of the review below. The choice of implemented models was based on an ambition to cover several different classes of models, but was also limited by the fact that many of the papers did not provide enough details to allow implementation.

\section{Models of driver behavior in collision situations}

In this section, driver models will be presented, following a rough taxonomy based on the main aspect of collision avoidance behavior addressed by the model in question. The first two subsections will be devoted to models with an emphasis on avoidance by either braking alone or steering alone, respectively. Then, models focused on the interplay of braking and steering will be covered. Thereafter, models will be described that emphasize how driver states and driver characteristics affect near-collision behavior. The last subsection will review papers in which a main topic of discussion has been the simulation platforms used for studying collision situations.

When providing model equations, a consistent notation will be used for recurring mathematical quantities, thus often departing from the exact symbols used by the original authors.

\section{Avoidance by braking}

Two main classes of deceleration-related driver models may be discerned: Those that do not take the concept of satisficing into account, and those that do. A defining characteristic of the former class is that these models will react to an obstacle at arbitrarily long distances. Below, the two classes will be reviewed separately.

Many of the models introduced below were originally proposed within the context of large-scale agentbased traffic simulations, or microscopic simulations, where obstacles are generally lead vehicles, and where driver models are referred to as car-following models (Brackstone \& McDonald, 1999; Helbing, 2001).

Models assuming long-distance reactions to obstacles. A well-known longitudinal control model of this type is the car-following model by Gazis, Herman, and Rothery (1961), often referred to as the GHR model. It 
was not developed specifically to study collision situations, but is presented here since several more recent models, either based on the GHR model or similar to it, have been applied in such contexts. The main equation in the GHR model is:

$$
\ddot{x}_{F}(t)=\lambda \cdot \Delta \dot{x}_{\mathrm{ff}}\left(t-T_{R}\right)
$$

where $\Delta x_{\mathrm{ff}}(t)=x_{L}(t)-x_{F}(t)$, and $x_{L}(t)$ and $x_{F}(t)$ denote the positions along the road (increasing in the forward direction) of the fronts of the leading and following vehicles, respectively ${ }^{2}$. Dots denote differentiation with respect to time, $T_{R}$ is a time lag (or an apparent reaction time), and $\lambda$ is a sensitivity term defined as

$$
\lambda=a \frac{\dot{x}_{F}^{m}(t)}{\Delta x_{\mathrm{ff}}^{l}\left(t-T_{R}\right)}
$$

where $a, l$ and $m$ are constants.

Thus, a GHR driver aims to keep the same speed as the lead vehicle (Equation 1), and corrects for speed differences more swiftly at high speeds and low headway distances (Equation 2). Figure 2 illustrates the model's long-distance reaction in an LVS scenario (left panel), as well as the more realistic, delayed deceleration pulsetype response in an LVD scenario (right panel). Since the GHR model, in this most basic form, disregards the physical extensions of vehicles, it tends to $\Delta x_{\mathrm{ff}}=0$ in both scenarios, corresponding to the two vehicles overlapping completely (i.e. a negative headway distance).

Over the years, much research has been devoted to the study of real traffic data in order to find the best parameter settings for the GHR model, but without conclusive results (Brackstone \& McDonald, 1999; Ozaki, 1993). Sultan, Brackstone, and McDonald (2004) suggested that more realism could be obtained in scenarios involving lead vehicle accelerations and decelerations (such as LVD, but not LVS and LVM), by adding acceleration terms to Equation 1.

H. Yang and Peng (2010) instead expanded on a GHR-like model by taking into account a number of error-inducing behaviors, as well as stochasticity (unpredictability) of driver behavior, in order for crashes to be generated in their simulations. Their core longitudinal control model, derived from a large data set of driving in real traffic, can be written as

$$
\begin{array}{r}
\ddot{x}_{F}(t)=P\left(\Delta x\left(t-T_{R}\right)\right) \Delta \dot{x}\left(t-T_{R}\right)+ \\
C \times\left[\Delta x\left(t-T_{R}\right)-\dot{x}_{F}\left(t-T_{R}\right) \Delta T_{d}\right]
\end{array}
$$

where $P(\Delta x)$ is a cubic polynomial, $C$ a constant, and $\Delta T_{d}$ the driver's desired time headway. The first term on the right hand side may be compared to Equation 1, and the cubic polynomial $P(\Delta x)$ proposed by $\mathrm{H}$. Yang and Peng bears some resemblance to a $1 / \Delta x$ function, corresponding to a GHR model with $m=0$ and $l=1$. The remaining term (proportional to $C$ ) achieves time headway control. Stochasticity was introduced by drawing acceleration values from a probability distribution around the value determined by Equation 3, with variance determined by another polynomial in distance headway. The introduced error-inducing behaviors were (a) a Weber ratio (Gray, 2010, p. 263) limiting detection of small changes in range rate; (b) eyes-offroad behavior, modeled as variations in reaction time $\left(T_{R}\right)$; and (c) mind-off-road behavior, modeled as temporary increases in acceleration control variability.

In simulation, this model generated rear-end crashes at approximately twice the rate observed in accident statistics. Yang and Peng argued that this discrepancy could be due to an inability of Equation 3 to account well for highly critical collision avoidance behavior. The LVD scenario behavior shown in the right panel of Figure 2, obtained for the model as written in Equation 3 , without stochasticity and error-inducing behaviors, indicates that the model comes close to avoiding the collision, but features clear oscillations in the braking response. In the LVS and LVM scenarios, this implementation of the model is unstable, due to the time headway control (not shown in the left panel of Figure 2).

Other models in this general class have been proposed by Chung, Song, Hong, and Kho (2005), who suggested a new formulation for the $\lambda$ term of the GHR model, and Mehmood, Saccomanno, and Hellinga (2001), who based their model on system dynamics concepts. Kuge, Yamamura, Boer, Ward, and Manser (2006) parameter-fitted a model of this class to noncritical driving with and without a support system involving a haptic gas pedal, and used these two parameter settings to predict the impact of support system presence on a more critical LVD scenario.

In summary, this class of braking model has seen much application in collision avoidance contexts, and

\footnotetext{
${ }^{2}$ The definition of the longitudinal distance between vehicles differs between models. Most models use the distance between the front of the following vehicle and the back of the lead vehicle, denoted $\Delta x$. However, in the GHR model, the vehicles are pointlike so that, if the extension of the vehicles were to be taken into account in that model, the longitudinal distance would correspond to the front-to-front (or, equivalently, back-to-back) distance. Hence, for that model the notation $\Delta x_{\mathrm{ff}}$ is used.
} 

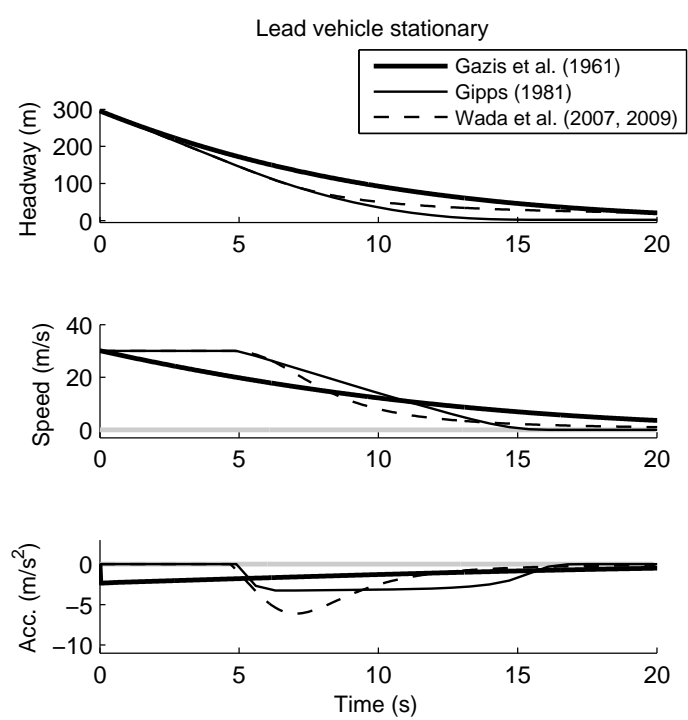
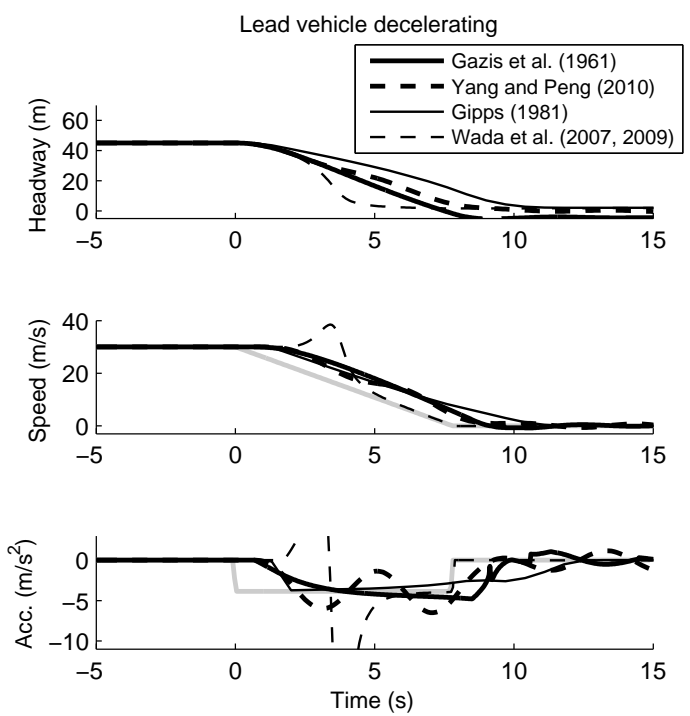

Figure 2. : Behavior of selected driver models focusing on braking. In both panels, the solid gray line shows behavior of the lead vehicle (length $l_{L}=4.5 \mathrm{~m}$ ). The left panel shows model behavior in a scenario with a stationary lead vehicle. At time $t=20 \mathrm{~s}$, headway values were $21 \mathrm{~m}$ and decreasing for the Gazis et al. (1961) and Wada et al. $(2007,2009)$ models, and the Gipps (1981) model was stable at $2 \mathrm{~m}$. The right panel shows model behavior in a lead vehicle decelerating scenario, with initial time headway of $1.5 \mathrm{~s}$ and constant $0.4 \mathrm{~g}$ lead vehicle deceleration starting at time $t=0 \mathrm{~s}$ and ending at full stop. At time $t=15 \mathrm{~s}$, headway values were $-4.5 \mathrm{~m}$ for the Gazis et al. model, $-0.4 \mathrm{~m}$ for the Yang and Peng (2010) model (here simulated without stochasticity and error-inducing behaviors), and $2 \mathrm{~m}$ for the Gipps and Wada et al. models. Parameters for the Gazis et al. model were: $T_{R}=0.7 \mathrm{~s}$, $a=1.1, l=1$, and $m=0.9$ (from Ozaki, 1993). Parameters for the Yang and Peng model were: $T_{R}=0.7 \mathrm{~s}, C=1$, $\Delta T_{d}=1.5$ s. $P(\Delta x)$ was estimated from their Figure 3. Parameters for the Gipps and Wada et al. models were as proposed in the corresponding papers.

the work of H. Yang and Peng (2010) is especially notable. However, thorough validation on real accident data is lacking so far. Furthermore, the inherent inability of these models to generalize to less critical scenarios, where a real driver may not always initiate braking as quickly as possible, may make them less useful in applications where both normal and critical collision avoidance must be simulated.

Delayed constant deceleration models. An additional set of models, which can be considered a subclass to the one discussed above, is delayed constant deceleration models. Here, this term is used when referring to models of the following general type, which has seen much use in previous research:

Starting at a (reaction) time $T$ after a stimulus $S$, the driver applies a constant deceleration $D$.

Such a model approximates the behavior of the GHR model in the critical LVD scenario (cf. the right panel of Figure 2), and shares that model's limitations with respect to less critical situations.

The most frequent context of application for this type of model has been the study of active safety warning systems, especially forward collision warning systems. Computer simulation with such a model, with the active safety warning constituting the stimulus $S$, has been used for optimizing system parameter settings, and to make predictions on potential traffic safety benefits on a societal level (T. Brown et al., 2001; Fitch et al., 2008; Krishnan, Gibb, Steinfeld, \& Shladover, 2001 and also Sugimoto \& Sauer, 2005, although the model used in that case was slightly more advanced). Other uses of this type of model has been assessment of safety impact of in-vehicle information systems, with $S$ being the first glance back towards the road after a lead vehicle has begun deceleration (Smith, Chang, Cohen, Foley, \& Glassco, 2005), development of road geometry design guidelines, with $S$ being the sudden appearance of an unexpected obstacle (Fambro, Fitzpatrick, \& Koppa, 2000), and a study of accident causation mechanisms, 
with $S$ representing the establishment of an initial collision course (Davis, 2007; Davis \& Swenson, 2006). McMillan, Christiaen, and Stark (2001) also relied on this general account of collision avoidance behavior for estimating the collision probability inherent in a given empirically observed rear-end situation, but instead of defining $S$ explicitly they varied brake initiation timing and deceleration magnitude around the empirically observed values.

Indeed, in all of the research cited above except that by Fambro et al. (2000), varying at least one of the quantities $T$ and $D$ was part of the approach. Often, the initial kinematics of the vehicles involved was also varied. In all cases except one (T. Brown et al., 2001), parameter variation was introduced using probability distributions, taken from previous literature or from a data set used in the corresponding research project. Another common factor in much of the abovementioned research has been the use of the driver model in analysis of recorded driving data, to answer what if? types of questions, such as: what if a forward collision warning system had been present in this situation? (Fitch et al., 2008; Sugimoto \& Sauer, 2005), what if a lead vehicle had suddenly braked during the performance of the in-vehicle secondary task? (Smith et al., 2005), or what if this traffic situation would have looked slightly different? (Davis \& Swenson, 2006; McMillan et al., 2001).

Although the simplicity of this type of model may rightfully raise questions of validity (for instance regarding the inability of satisficing behavior), it may nevertheless serve as an example of how also very basic quantitative driver models can be put to some use in the study of traffic safety. In some of the research cited above, the resulting overall model dynamics was even tractable analytically, so that actual simulation was not needed (Davis, 2007; Fambro et al., 2000; Krishnan et al., 2001; McMillan et al., 2001).

Models assuming timed brake application. A satisficing driver approaching an obstacle may be assumed to exhibit a safety margin-related, timed transition from a non-decelerating state to a decelerating state. Longitudinal driver models have been proposed that try to capture such a phenomenon. In the much cited model by Gipps (1981), the mode transition in LVS, LVM, and LVD scenarios, as exemplified in Figures 2 (left panel) and 3, occurs when the vehicle speed prescribed by the equation

$$
\begin{array}{r}
\dot{x}_{F}(t)=b_{F} T_{R}+\left[b_{F}^{2} T_{R}^{2}-b_{F}\left(2\left[\Delta x\left(t-T_{R}\right)-s_{L}\right]\right.\right. \\
\left.\left.-\dot{x}_{F}\left(t-T_{R}\right) T_{R}-\frac{\dot{x}_{L}\left(t-T_{R}\right)^{2}}{b_{L}}\right)\right]^{1 / 2}
\end{array}
$$

falls below the initial cruising speed of the following vehicle. This equation, as written above, is difficult to interpret, but the assumption from which it is derived, using basic Newtonian mechanics, is clear: The following vehicle driver is assumed to adjust speed to a value such that, if the lead vehicle should suddenly brake with an assumed maximum deceleration $b_{L}<0$, the following vehicle driver will be able to avoid a collision without exceeding the own preferred maximum deceleration $b_{F}$, as long as the actual reaction time does not exceed a safety reaction time $1.5 T_{R} . s_{L}$ is the effective size of the lead vehicle, "the physical length plus a margin into which the following vehicle is not willing to intrude" (Gipps, 1981, p. 106). It may be noted that, in contrast to the GHR model, which predicts vehicle acceleration at each time step, the Gipps model operates directly on the vehicle speed. Gipps demonstrated, however, that in his model the effective deceleration in a simulation time step will never exceed $b_{F}$.

Figure 2 shows that in an LVD scenario, the Gipps model replicates the delayed constant deceleration type of behavior already seen for non-satisficing models, but manages to avoid the collision and to stop in a controlled manner. In an LVS scenario, the model generates a clearly identifiable, timed brake initiation. Interestingly, Figure 3 indicates that inverse TTC at brake initiation, as predicted by the Gipps model, exhibits a similar dependence on following vehicle speed as that observed by Kiefer et al. (2005) in their test track data, although with a different effect of lead vehicle speed (higher values of inverse TTC for LVS than for LVM, rather than the other way around). Further support for the Gipps model was provided by K. Lee and Peng (2004), in their benchmark comparison of carfollowing models measuring model performance when fitting normal driving and non-critical approach sequences. K. Lee and Peng (2004) and Peng (2002) also proposed a slight modification to the Gipps model that, however, does not seem to have a large impact on the types of scenarios studied here.

In its original formulation, the Gipps model will never lead to actual crashes in simulation, which is clearly a limitation in the study of near-crash and crash events. However, as in the case of the GHR model, some researchers have adapted the Gipps model to 
study accident situations. A crash-inclusive variation to the model was proposed by Hamdar and Mahmassani (2005), with the aim of mimicking driver behavior in traffic situations of general panic (e.g. during evacuations due to natural disasters or similar events). Another variation, aimed at driving in more normal traffic conditions, was proposed by Xin, Hourdos, Michalopoulos, and Davis (2008). Their approach was based on complementing the basic Gipps model with a number of insights from psychology (not dissimilar to what H. Yang \& Peng, 2010 did with their GHR-like model). A visual scanning interval, mimicking a divided attention to driving, was introduced, and at each scan a new target speed according to Equation 4 would only be set if changes in relative position or motion exceeded perceptual thresholds (Weber ratios), or if the lead vehicle deviated from a desired time headway $\Delta T_{d}$ by more than a certain fraction (headway satisficing). It was demonstrated that the model could be parameter-fitted to sequences of real driving from both a data set of normal, non-critical traffic, and a data set of six crashes and four near-crashes, involving a total of 54 vehicles on a high crash-rate section of a US freeway.

Another general means of introducing satisficing in a behavior model is to include concepts from fuzzy logic (Zadeh, 1965), where fuzzy states are typically defined as intervals for involved state variables, and control actions may be modeled as occurring only once deviation from a non-action state becomes large enough. Steigerwald (2002) carried out a simulation study of the safety effects of collision warning systems, using the fuzzy logic car-following model of McDonald, Wu, and Brackstone (1997). This model was driven by fuzzy rules such as, for example if distance divergence is Too Far and relative speed is Closing then the driver's response is No Action (keep current speed). Steigerwald observed crashes in his simulations, and found reductions in crash rate when driver response to collision warnings was included, modeled as a decreased reaction time setting in the car-following model.

Finally, the following quantity, which has been used in two separate models of braking behavior, will be considered:

$$
K_{d B}= \begin{cases}10 \log _{10}(K) \operatorname{sign}(-\Delta \dot{x}) & \text { if } K \geq 1 \\ 0 & \text { if } 0 \leq K<1\end{cases}
$$

where $K=\left|4 \cdot 10^{7} \cdot \Delta \dot{x} / \Delta x^{3}\right| \mathrm{km} \mathrm{h}^{-1} \mathrm{~m}^{-3}$ (note the nonSI unit, used by the original authors, for relative speed). This quantity was defined by Wada, Imai, Tsuru, Isaji, and Kaneko (2007), based on a retinally oriented account reminiscent of that of D. Lee (1976). When a collision course is established, $K_{d B}$ will rise from zero, and will reach higher values as the vehicle approaches collision. Wada, Imai, et al. (2007) found that in less critical situations, driver control of deceleration could be well described as a strategy in which $\mathrm{d} K_{d B} / \mathrm{d} \Delta x$ is held constant. Furthermore, they found that brake initiation timing could be modeled as occurring once a lead vehicle speed dependent variant of $K_{d B}$ surpassed a threshold (Wada, Doi, et al., 2007; Wada, Hiraoka, \& Doi, 2009). Figure 3 indicates that this brake initiation model is qualitatively similar to what the Kiefer et al. (2005) and Gipps (1981) models predict, although with no visible difference between LVM and LVS. In an LVS scenario the behavior of the model by Wada, Imai, et al. (2007) and Wada et al. (2009) is also qualitatively similar to that given by the Gipps model (Figure 2, left panel), but with stronger and more brief deceleration, whereas the right panel of Figure 2 suggests that a strategy in which $\mathrm{d} K_{d B} / \mathrm{d} \Delta x$ is kept constant is not realistic in the more critical LVD scenario, as indicated by the authors themselves.

Akita, Inagaki, Suzuki, Hayakawa, and Tsuchida (2007) incorporated the $K_{d B}$ quantity in their piecewise linear auto-regressive exogenous inputs (PWARX) model of driver speed keeping. By use of clustering algorithms on data in the $\left(K_{d B}, \Delta \dot{x}, \Delta x\right)$ space from a small simulator study $(n=2)$, four speed keeping modes, of which one was collision avoidance, were identified and separated. For each of these modes, a separate ARX model, each on the form:

$$
p(k+1)=a K_{d B}(k)+b \Delta \dot{x}(k)+c \Delta x(k)+d p(k)
$$

was fitted to the data. In this equation, $k$ denotes simulation time step, $p$ is the pedal input, and $a, b, c$, and $d$ are driver-dependent constants. Judging by the figures provided by Akita et al. (2007), it however seems as if braking events in their dataset were sparse, and possibly not highly critical.

In summary, this class of braking model may be more generally applicable than other models, since it is able to exhibit satisficing behavior in less critical scenarios, such as LVS and LVM. Figure 3 points to a possible convergence in this respect between some of the models. Furthermore, the simulated behavior of the Gipps (1981) model seems more stable than the simulated non-satisficing models. Whereas validation on real accident data is generally missing also for this model class, Xin, Hourdos, Michalopoulos, and Davis (2008) have provided a commendable exception. Further details on their work, including calibrated values 


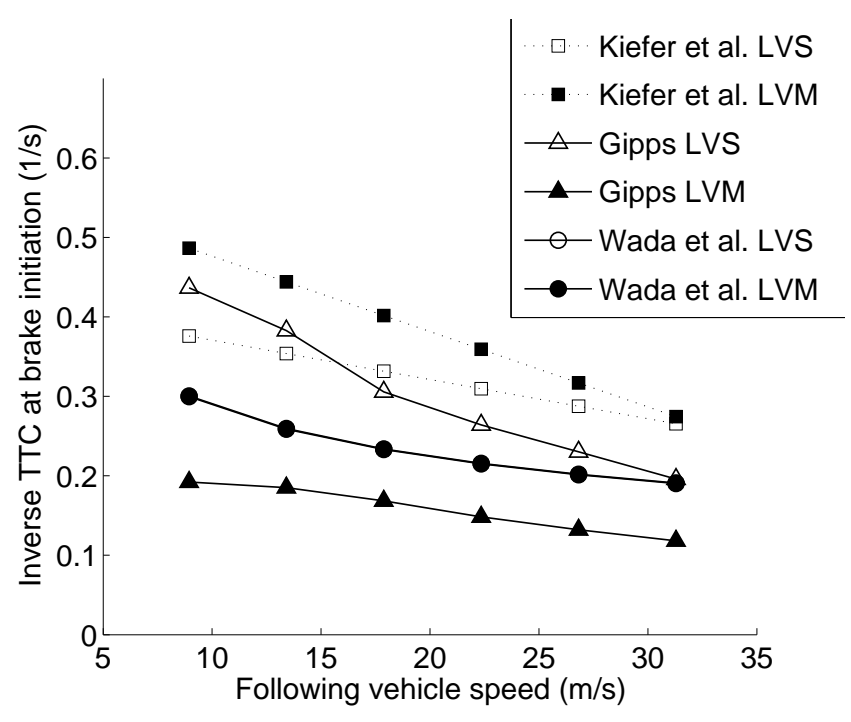

Figure 3. : Inverse time to collision at brake initiation, as a function of following vehicle speed, in the lead vehicle stationary (LVS) and lead vehicle moving (LVM) scenarios, for the Gipps (1981) and Wada et al. (2009, Equation 9) models, respectively, as compared to the results of Kiefer et al. (2005). In both simulated scenarios, the initial following vehicle speed was $30 \mathrm{~m} / \mathrm{s}$, and in the LVM scenario the lead vehicle speed was 15 $\mathrm{m} / \mathrm{s}$. The two curves for the Wada et al. model are not identical, but are close enough to appear overlapping in this figure.

for model parameters, can be found in (Xin, Hourdos, \& Michalopoulos, 2008).

\section{Avoidance by steering}

Few driver models are specifically designed for collision avoidance by steering. However, many models of steering have been benchmarked on rapid evasive maneuvers that mimic collision avoidance situations, such as the ISO double lane change (ISO, 1999). Here, the main objective has typically not been to create models that accurately replicate human behavior in accident situations. Rather, research has been more focused on finding models that perform well in terms of path tracking and stabilization. Nevertheless, since detailed steering models are important in some collision-related application contexts, all identified steering models tested on rapid evasive maneuvers have been included in this review, and their control performance will be reported below. A later section will then describe how some models have been tuned for sub-optimal performance, to account for inter-driver behavior variability in colli-
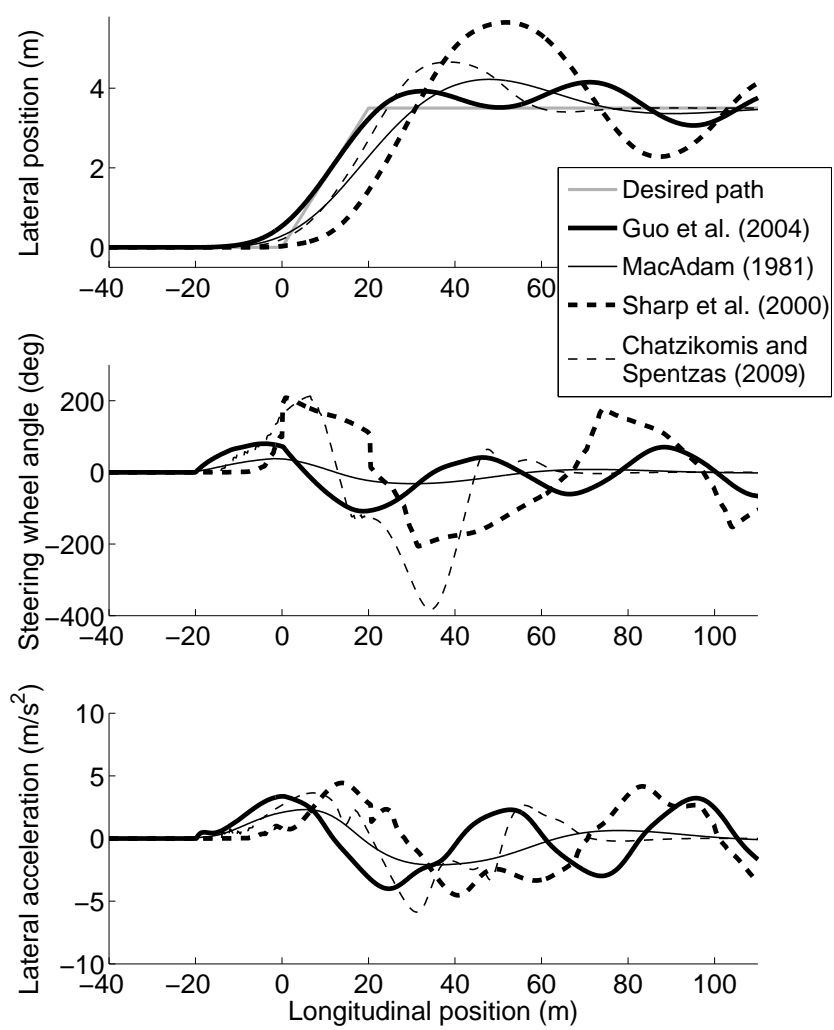

Figure 4. : Steering behavior of selected driver models carrying out a $20 \mathrm{~m}$ single lane change, at $20 \mathrm{~m} / \mathrm{s}$. Parameters for the models were chosen as proposed in the corresponding papers. Among the parameter sets proposed by MacAdam (2001), the following was adopted: A preview time of $1.3 \mathrm{~s}$, a response delay of $0.2 \mathrm{~s}$, and 10 preview points.

sion avoidance.

Traditionally, driver models with steering capabilities have been based on classical control theory (Jürgensohn, 2007). Even though other types of models have been developed recently, most of the steering models are still based on, or contain elements from, control theory. Typically, the input to such models is a desired path containing the desired lateral road position over time. In order to correct deviations from this desired path, the steering models output one of the following quantities: (a) The steering wheel angle, (b) the vehicle steering angle, or (c) the lateral acceleration.

When simulating models that operate on steering angles rather than directly on lateral acceleration, a ve- 
hicle model must be incorporated in the simulation. For our simulations (see Figure 4) we used the simulation environment of Benderius, Markkula, Wolff, and Wahde (2011), and implemented the vehicle dynamics model of Thommyppillai, Evangelou, and Sharp (2009), with parameters as specified in Appendix B.

Models using path preview. In order to predict deviations before they occur, driver models often use path preview. The simplest form is single point preview, where the expected deviation in, for instance, lateral position or heading angle is measured at a single point located a distance $S_{p}$ in front of the vehicle. The distance $S_{p}$ is often defined as a function of a constant preview time $T_{p}$ as

$$
S_{p}(t)=\dot{x}(t) T_{p}
$$

where $\dot{x}(t)$ is the longitudinal speed of the vehicle.

Reński (2001) and M. Lin, Popov, and McWilliam (2003) independently of each other proposed two similar single point preview models, in which the vehicle steering angle $\delta(t)$ is given by

$$
\delta(t)=K \epsilon\left(t-T_{R}\right)
$$

where $\epsilon(t)$ is the angle between the heading of the vehicle and the preview point, $T_{R}$ the driver reaction time, and $K$ a gain constant ${ }^{3}$. Both Reński and M. Lin et al. showed that this driver model was capable of carrying out a double lane change maneuver, and studied model behavior for varying parameter settings. Furthermore, Reński optimized model parameters to reproduce the recorded trajectory of a real driver.

Guo, Ding, Zhang, Lu, and Wang (2004) also used single point preview in their preview-follower model, originally proposed by Guo and Fancher (1983). Within this model, the current acceleration, velocity, and position of the vehicle are used in order to predict the lateral error at time $t+T_{p}$. Then, the steering wheel angle required to correct this previewed error is calculated, assuming a simple vehicle model. Guo et al. (2004) compared data from a simulator study where drivers carried out a double lane change maneuver, with model output for a preview time of $1.4 \mathrm{~s}$, and found good agreement. The same model was used in a double lane change also by Gao, Zheng, Guan, and Guo (2002). Figure 4 suggests that when parameterized as proposed by Guo et al. (2004), this model can manage a rapid single lane change very well, although with some problems regaining stability afterwards.

Based on the preview-follower model, Zhuang, Yu, and $\mathrm{Li}$ (2005) applied an artificial neural network in order to calculate optimal preview times for a variety of different test tracks at different vehicle speeds. It was found that optimal preview times (for the model used) were in the range from 1.1 to $1.3 \mathrm{~s}$, and that higher speeds required slightly longer preview times (suggesting that Equation 7 should, in fact, be somewhat nonlinear).

Overall, it may be noted at this point that the use of a desired path in combination with some form of path preview may raise questions regarding model validity in real collision situations. One could argue that this type of preview, as well as some of the even more advanced control theory practices reviewed below, imply that the modeled driver plans ahead in a more controlled fashion than what may be the case in accident situations. Alternative standpoints are also possible, however, and the topic will be considered further in the discussion.

Multi-level models. Donges (1978) provided the first example of another class of driver models, the two-level driver models, which typically include path preview as discussed above. The two levels are called anticipation and stabilization (the latter also compensation or guiding). At the anticipation level, steering is estimated in an open-loop manner based on the curvature of the previewed desired path and a simplified vehicle dynamics model. The vehicle model is referred to as the internal vehicle model, representing the driver's understanding of the vehicle. Deviations from the desired path can, for instance, occur as a result of system noise or simplifications in the internal vehicle model. At the stabilization level, the model compensates for such deviations in a closed-loop manner.

A recent two-level model was introduced by Edelmann, Plöchl, Reinalter, and Tieber (2007). In this model, a nonlinear vehicle dynamics model is locally linearized and used at the anticipation level. By using two preview points in front of the vehicle (in order to anticipate changes in curvature) a steering wheel angle estimate can be calculated.

Plöchl and Lugner (2000) introduced a three-level driver model. If large local path deviations occur, the third level can temporarily override anticipation and stabilization in order to steer towards the desired path as quickly as possible without using path preview. This approach is interesting especially in relation to the potential concerns raised above, regarding the use of preview in models of critical collision avoidance.

\footnotetext{
${ }^{3}$ It may be noted, however, that M. Lin et al. (2003) themselves referred to their model as a two-level model, as discussed in the next section.
} 
Models using optimization over a preview horizon. Another class of model determines a steering response by optimizing over a preview horizon (also referred to as the preview interval). This relates to the control theory concept of optimal control (see e.g. Kleinman, Baron, \& Levison, 1970; Vinter, 2010). Here, the term optimal does not imply that these models cannot exhibit satisficing behavior. Optimization criteria can be defined so as to manage a trade-off between, for example, path deviation and steering effort, which would typically be referred to as satisficing.

An early driver model optimizing over a preview horizon was introduced by MacAdam (1981). The model determines, in each time step, the vehicle steering angle that minimizes a path deviation cost function over the preview horizon. The top panel of Figure 4 shows good tracking performance for this model, even with comparably small steering wheel inputs (middle panel). In a later version of this model (MacAdam, 2001, 2003), the original linear internal vehicle model was replaced by a non-linear one, improving the performance of the driver model in situations close to the limits of the vehicle's capabilities. The extended model also offers the possibility to have the preview time varying in magnitude depending on upcoming road geometry. When using this feature in a double lane change, the preview time was shown to vary within a range from 0.6 to $2.0 \mathrm{~s}$.

Peng (2002) developed a driver model that extends MacAdam's original model (1981) with a more general cost function, as well as the capability of updating the internal vehicle model during operation, something that is often referred to as vehicle adaptation. These extensions were later shown to improve control performance in a double lane change maneuver Ungoren and Peng (2005).

Prokop (2001) and Butz and von Stryk (2005) proposed models with a variety of terms in their cost functions, including satisficing-related terms aimed at limiting lateral accelerations. These two models differ from the other reviewed optimal control models in that they are two-level models, first determining a desired path by optimization over the preview interval, and then applying stabilization control to follow the optimized trajectories. Butz and von Stryk provided examples of their model's behavior in a double lane change maneuver, for a number of different weightings of their optimization criteria. A similar model can also be found in (Vögel, von Stryk, Bulirsch, Wolter, \& Chucholowski, 2003).

Yoshida, Shinohara, and Nagai (2008) employed an optimization method similar to the one used by
MacAdam to derive open-loop steering interventions for use in an active safety system, aimed at achieving automatic collision avoidance.

Models using multi-point preview. Models optimizing over a preview horizon show good stabilization capabilities in relation to rapid maneuvers (see e.g. Figure 4). However, optimizing over a interval may, depending on sampling rate and optimization method, be computationally intensive. An alternative approach is to use multi-point preview, in which the driver model uses a discrete number of points in front of the vehicle for its tracking behavior. Typically, the points are individually weighted and positioned at fixed preview times.

The model by Sharp, Casanova, and Symonds (2000) uses a multi-point preview control scheme, where points are positioned at fixed preview times in front of the vehicle. The vehicle steering angle $\delta(t)$ is given by

$$
\delta(t)=K_{\psi} \psi_{e}(t)+K_{1} y_{e_{1}}(t)+\sum_{i=2}^{n} K_{i} y_{e_{i}}(t)
$$

where $\psi_{e}(t)$ is the heading error compared to the tangent of the desired path, $y_{e_{1}}(t)$ the vehicle's lateral deviation, $y_{e_{i}}(t)$ the lateral deviation of the preview points, and $K_{\psi}$, $K_{1}$, and $K_{i}$ are gain constants. The model was originally intended for race track applications, but was used by Wenzel, Burnham, Williams, and Blundell (2005) for studying the utility of a stability support system in a double lane change maneuver. The large overshoot seen in our simulations (Figure 4) is probably due to the fact that Sharp's parameterization is tuned for race car dynamics.

In the multi-point model by Thommyppillai et al. (2009) a non-linear vehicle model is linearized for different values of the vehicle speed and the front axle slip ratio. An adaptive control strategy, where control gains are derived depending on vehicle properties, is then determined by using the linearized vehicle model. In the same paper, the authors compared the adaptive control strategy with a fixed gain strategy, and found that the model with adaptive gains showed significantly better tracking performance.

Another multi-point driver model, which has been used in a double lane change, was proposed by Chatzikomis and Spentzas (2009). The model uses a combination of two error measures: (a) Errors in heading angle, and (b) errors in lateral position. A vehicle steering angle is then calculated using a weighted sum of both errors, and an adaptive control strategy based on longitudinal speed. By determining error weights using stochastic optimization, Chatzikomis and Spentzas 
found that, for their model, heading angle errors were a more important input than lateral position errors. Figure 4 indicates that this model achieves good tracking performance, despite large steering magnitudes.

Other models of steering. Some models will now be described that do not fit into the model classes outlined above. Gao and Jiang (2009) proposed a model in which path planning is implemented. At any instant in time, all feasible trajectories are determined based on the current vehicle state, steering limit, and lane boundaries. The best trajectory is then chosen according to a safety index, based on the distance to the center line, and a maneuverability index, based on the required change in the lateral acceleration.

A piecewise polynomial steering model intended for obstacle avoidance was proposed by Kim et al. (2005). The model consists of four modes, each modeled in the form

$$
\delta_{S W}(t)=a \Delta x(t)+b \Delta \dot{x}(t)+c \Delta y(t) \quad \text { if } d \leq \Delta x(t)<e
$$

where $\delta_{S W}(t)$ is the steering wheel angle, and $\Delta x(t)$ and $\Delta y(t)$ the longitudinal and lateral distances, measured relative to the obstacle. The parameters $a, b, c, d$, and $e$ are constants which Kim et al. determined from driving data. Each mode represents a specific steering behavior that is activated depending on the longitudinal distance to the obstacle; see Equation 6 for a similar model of driver braking behavior.

A driver model based on a neural network was proposed by Y. Lin, Tang, Zhang, and Yu (2005). The model has two outputs, steering wheel angle and steering wheel rate, and uses seven inputs, one of which is the lateral displacement from the desired path, another is the lateral displacement of a preview point, and the rest are vehicle state properties. Y. Lin et al. (2005) tested the model, by first training the network on driving data, in various steering maneuvers.

A steering model that differs from the models previously discussed in this section, in the sense that it models a purely open-loop response, was introduced by Araszewski, Toor, Overgaard, and Johal (2002). It was developed for the reconstruction of single lane change maneuvers in accident situations. For a given vehicle speed and given longitudinal and lateral lane change distances it generates a sine or triangle wave steering wheel response. The authors did not report on any use or validation of the model in reconstruction of actual accidents.
Avoidance by a combination of braking and steering

In several of the papers cited in the previous section (e.g. Butz \& von Stryk, 2005; Chatzikomis \& Spentzas, 2009; Guo et al., 2004; MacAdam, 2001; Prokop, 2001), models of braking were also proposed in order to adjust speed in normal driving conditions, for example in curve negotiation. This section will focus specifically on models that have been used for simulating the combined use of braking and steering in collision avoidance.

The driver model proposed by Jurecki and Stańczyk (2009) is capable of both steering and braking, and was designed specifically for collision avoidance at intersections. The braking of the model is defined as

$$
a_{b}(t)+K_{1} \dot{a}_{b}(t)=K_{2} \Delta y\left(t-T_{R_{b}}\right)+K_{3} \frac{\Delta \dot{x}(t)}{\Delta x(t)}
$$

where $a_{b}(t)$ is the vehicle deceleration, $\Delta x(t)$ and $\Delta y(t)$ the longitudinal and lateral distances to the conflicting car (driving in a direction perpendicular to the modeled driver's vehicle), $T_{R_{b}}$ the braking reaction time, and $K_{1}$, $K_{2}$, and $K_{3}$ are constants. The steering of the model is defined as

$$
\delta(t)+K_{4} \dot{\delta}(t)=K_{5} \Delta y\left(t-T_{R_{s}}\right)
$$

where $\delta(t)$ is the vehicle steering angle, $T_{R_{s}}$ the steering reaction time, and $K_{4}$ and $K_{5}$ are constants. In order to find suitable values for the model constants, Jurecki and Stańczyk used driving data acquired from a test track experiment.

By conducting an experiment where subjects were asked to drive around a test track, Yamakado and Abe (2008) found a relation between lateral (steering) and longitudinal (braking) accelerations during driving. They defined this relation, in the Laplace frequency domain (frequency variable $s$ ), as

$$
a_{x}(s)=-\operatorname{sign}\left(a_{y}(s) \dot{a}_{y}(s)\right) \frac{K \dot{a}_{y}(s)}{1+T_{d} s}+a_{x_{0}}(s)
$$

where $a_{x}(s)$ is the longitudinal acceleration, $a_{y}(s)$ the lateral acceleration, $K$ a gain constant, $T_{d}$ a delay time, and $a_{x_{0}}(s)$ a model input denoting the working point of the longitudinal acceleration. In practice, this means that longitudinal acceleration will, with a delay determined by $T_{d}$, tend to (a) $a_{x_{0}}$, if lateral acceleration is constant; (b) a more negative value than $a_{x_{0}}$, if lateral acceleration is increasing (to either left or right); or (c) a more positive value than $a_{x_{0}}$, if lateral acceleration 
is decreasing. Yamakado and Abe complemented this model with the preview-follower model of Guo et al. (2004), and found good agreement between their simulations and single lane change data from a test track.

In another class of driver models, generally exhibiting satisficing behavior, control inputs are calculated based on road or obstacle boundaries rather than (as for the steering models discussed so far) on a desired path (predefined or optimized). For instance, Gordon and Magnuski (2006) introduced a driver model that, by treating all navigation as collision avoidance, is capable of both braking and steering. According to the current state of the vehicle dynamics, the model estimates the point where the vehicle would cross the lane boundary. By applying appropriate control input, the driver shifts the estimated crossing point further down the road. Similar models, based on fuzzy logic, navigate as a result of always trying to keep lane boundary distances within safe margins (El Hajjaji \& Ouladsine, 2001; Zeyada, El-Beheiry, El-Arabi, \& Karnopp, 2000). Zeyada et al. showed that their relatively simple model manages both to negotiate a sharp turn, and to avoid an obstacle.

Gordon and Best (2006) introduced another model that does not require a desired path. Based on road geometry, a velocity vector field is determined for the drivable area. At each point in the vector field, the desired velocity, acceleration, heading, and yaw rate are determined. The steering angle and the deceleration are then derived using simple control strategies.

Sugimoto and Sauer (2005) carried out what-if simulations of reconstructed rear-end accidents to estimate the potential benefit of a collision avoidance support system. Their driver model responded to collision warnings either by open-loop evasive steering behavior(implemented as one period of a sine wave), by an open-loop evasive braking behavior (implemented as a delayed constant deceleration), or by a combination of the two. However, although the simulated scenarios were based on actual accidents, the authors provided only limited empirical support for the driver model itself.

\section{Effects of driver states and characteristics}

Next, models will be discussed that emphasize variability in near-collision behavior, due to (a) visual distraction, (b) driving skill and style, and (c) other driver states and characteristics.

Effects of visual distraction. Several researchers have put modeling emphasis on the collision-related effects of glances directed away from the forward roadway, e.g. to mirrors or in-vehicle displays. Two phenomena which have been recurrently addressed in this context are (a) visual allocation strategies, determining how a driver will control eyes on/off road behavior; and (b) off-road glances delaying driver reactions to a potential forward collision threat at least until gaze is again on the road ahead.

Smith et al. (2005), studied the safety effects of invehicle information systems, by replaying in simulation actual recorded vehicle following sequences, including on/off road glance data. Thus, in this case, there was no need for a separate model of visual allocation, only the stimulus-reaction type braking response previously discussed in the section on delayed constant deceleration models.

Delorme and Song (2001) proposed a model of driver behavior for use in traffic microsimulation. The model was based on structuring the driving task into separate driving schemata, such as following and overtaking. In the context of this review, the main importance of this model was the proposed approach of relating the occurrence of speedometer glances and side glances to the currently active driving schema, and the use of externally scripted commands to trigger glances relating to in-vehicle secondary tasks. Deceleration and braking behavior, available when gaze was on-road, was then triggered by TTC thresholds and was implemented as linear control of range and range rate. No validation or calibration on critical driving situations was provided, however.

In the attention-based rear-end collision avoidance model (ARCAM) of T. Brown, Lee, and McGehee (2000), visual allocation was driven by an uncertainty regarding the collision potential with respect to a lead vehicle. In ARCAM, uncertainty increases as a function of time during off-road glances, and on-road glances are triggered when uncertainty reaches a threshold level. Deceleration in response to collision situations is then delayed by an expectancy-dependent reaction time, and deceleration magnitude is set as a function of the collision potential quantity, in closed-loop control (not defined in detail by the authors). ARCAM was validated to some extent on simulator data, by T. L. Brown, Lee, and McGehee (2001), and was put to practical use by J. D. Lee et al. (2002). They used the model to extrapolate from data found in a simulator study on visual distraction and collision warnings, thus demonstrating a potential benefit of computer simulation as a complement to tests with real drivers in the loop. 
Effects of driving skill and driving style. Some authors have studied how to adapt driver behavior models to represent varying levels of driver skill, as well as different driving styles. This has been done, for example, in the context of simulation-based estimation of vehicle handling properties (Data, Pascali, \& Santi, 2002), and to support arguments on how to adapt the dynamics of an articulated vehicle to suit different driver skill levels (X. Yang, Rakheja, \& Stiharu, 2001).

All of the reviewed models in this group have been control-theoretic models of avoidance by steering, similar to those discussed earlier in this paper, and all have been applied to double lane change maneuvers: X. Yang et al. (2001) used a single-point preview model, extended with provisions for stabilization of truck-trailer articulation. Data et al. (2002) used a two-level model. The model of Noh, Jung, Choi, and Yi (2007) performed optimization over a preview horizon. Erséus (2010) adopted the high-level architecture of MacAdam (2003), but proposed new formulations for the individual sub-modules. Irmscher and Ehmann (2004) used the driver component of a commercial simulation environment, but did not describe the driver model in full detail ${ }^{4}$.

Among these authors, only Data et al. (2002) did not discuss any specific dimensions of driving skill or style. They varied the preview time and control gain parameters of their model, resulting in variations in steering wheel behavior that were qualitatively similar to what had been observed for three drivers on a test track.

The other authors listed above have all addressed driving skill in the parameterizations of their models. The strongest common denominator has been the treatment of the preview time parameter, which has consistently been set to higher values when representing more skilled drivers. In the case of X. Yang et al. (2001), this was also complemented with a lower control gain setting. Furthermore, all of these authors have modeled less skilled drivers as having slower and more inexact steering responses, although achieved in slightly different ways by different authors. Erséus (2010) and Irmscher and Ehmann (2004) also increased the accuracy of the internal vehicle model as a function of increasing skill. In terms of validation, Noh et al. (2007) and Erséus (2010) were alone in comparing their results with real driving data, from a test track and a driving simulator, respectively.

Additionally, Irmscher and Ehmann (2004) varied model parameters to capture behavior of aggressive drivers. These were hypothesized to over-estimate the cornering abilities of their vehicle, and to use shorter preview distances and less smooth desired paths. The combination of low skill and high aggressivity was shown to result in a driver model that was prone to control loss in the double lane change maneuver.

Effects of other driver states and characteristics. In addition to the aspects considered above, several other driver states and characteristics have been modeled and simulated in the context of collision avoidance. Some of these contributions come from Salvucci and colleagues, who use the cognitive architecture ACT-R (atomic component of thought-rational) to model driver behavior in general (Salvucci, 2006). ACT-R is modular, and at its core are a number of buffers, written to and read from by the various modules and by central IFTHEN type production rules, firing in series at frequencies up to a maximum rate (typically one production rule every $50 \mathrm{~ms}$ ), thus acting as a central bottleneck for cognition. This cognitive architecture has been used to model and reproduce a large number of experimental results (Anderson \& Lebiere, 1998).

In two related papers, Salvucci (2002) and Salvucci and Taatgen (2008) extended the ACT-R driver model to account for cognitive distraction due to a secondary task where several words needed to be kept in memory. In these contributions, both the primary driving task and the secondary cognitive task were modeled as requiring repeated firing of production rules in a central processing resource. Since these production rules fired serially, performance of the cognitive secondary task resulted in a general slow-down of driving-related processing in the model, which caused increases in reaction times to safety-critical lead vehicle braking events, as observed in a driving simulator experiment.

Salvucci, Chavez, and Lee (2004) modified the ACT$\mathrm{R}$ model of driving in order to study effects of old age on brake response, in a situation with risk of stop light violation, but without intersecting traffic (thus making the model a borderline case for inclusion in this review). Based on previous work on ACT-R and aging, they introduced a $13 \%$ slow-down of production rule firing rate, and were thus able to reproduce an interaction effect observed in a test track experiment: When driving with a visual-manual secondary task, brake reaction times of both young (25-36 years) and old ( $>55$ years) drivers increased, but the increase was significantly greater for the older drivers.

\footnotetext{
${ }^{4}$ Also MacAdam (2001), Prokop (2001), and Ungoren and Peng (2005) discussed, to some extent, how to parameterize their steering models to account for effects of driving skill and style, but put less emphasis on this issue.
} 
A similar slow-down due to old age was introduced by B. Wang et al. (2002), in a control theoretic model due to Allen (1982), controlling lane position and heading to follow a predefined desired path. B. Wang et al. studied the behavior of this model for values of its neuromuscular delay parameter corresponding to simple task reaction times reported in the experimental literature (around $0.30 \mathrm{~s}$ and $0.45 \mathrm{~s}$, for young and old drivers, respectively, i.e. an increase of $50 \%$ with age). They also varied the amount of derivative control of the model, citing previous researchers who found such control to be less pronounced in older drivers. Their simulations predicted that aged drivers should perform worse (deviate more from the desired path, use wider steering movements, or a combination thereof) during a single lane change, especially on a low friction road surface, and that they would benefit from a proposed fourwheel steering control algorithm. These predictions were not validated on any actual driving data, however.

Age-related slowing of reaction time (by 20\%) was also included in the driver-vehicle simulation model by Delaigue and Eskandarian (2004), aimed at predicting total stopping distances of passenger cars in emergency braking. Based on previous literature, they proposed a non-driver-specific model of braking foot movement, triggered by a braking stimulus, but with a driverspecific reaction time delay. Expressions were provided for the mean and variance of this delay, as a function of driver age, driver gender, and degree of expectancy of the stimulus. However, the driver parts of their model were not subject to any validation.

\section{Simulation platforms}

A number of researchers have proposed entire platforms for simulation of traffic with collisions (Furukawa, Seki, \& Fujikawa, 2009; Kitaoka et al., 2009; Wood, Dumbuya, Zhao, Hill, \& Thomas, 2003; Yuhara \& Tajima, 2006). This is reminiscent of the high-level perspective adopted in the previously mentioned field of microscopic traffic simulation, although the driver models reviewed here differ markedly from the car-following models typically used in that field (see Brackstone \& McDonald, 1999; Helbing, 2001). None of the reviewed papers provided full specifications of the driver models, but strong common denominators can nevertheless be discerned, especially in terms of the adopted general model architectures, which are clearly inspired by information processing concepts (Wickens \& Hollands, 2000). In a first step, the proposed driver models perceive the traffic surroundings, and build a mental representation of it. Then, based on this rep- resentation, rule-based decisions on actions are made, and finally the actions are carried out, in terms of vehicle operation and control.

A majority of the models (Furukawa et al., 2009; Kitaoka et al., 2009; Yuhara \& Tajima, 2006) include explicit, probabilistic mechanisms for error generation, at all three stages of processing outlined above. The errors are introduced in order to have simulations generate accidents, with the purpose of estimating safety impacts of various active safety systems. Kitaoka et al. (2009) and Yuhara and Tajima (2006) also included basic on/off road visual distraction behavior. Wood et al. (2003) did not include any explicit accident causation mechanisms, but instead manually tuned decisionmaking of their driver models to reproduce a specific head-on collision event from real traffic.

Furthermore, Kitaoka et al. (2009) put a stronger emphasis than the others on driver characteristics and states, and Yuhara and Tajima (2006) were alone in including a specific evasive maneuvering mode, triggered by a TTC criterion. However, thorough validation on accident-related data from real traffic seems to be lacking for all models.

Garcia, Libreros, and Contreras (2008) proposed an infrastructure-oriented simulation platform, aimed at predicting risk of accidents due to visibility problems at skewed intersections.

\section{Discussion}

The discussion below will focus on three topics, namely (a) how to choose models for use in future applications, (b) potential areas for future model development, and (c) issues related to model validation and comparison. Some concluding remarks will also be made.

\section{Putting the reviewed models to use}

The reviewed papers show a wide range of applications of driver models in computer simulation. Notable examples of methodologies include the use of simulation for (a) identification of preferable designs for vehicles or infrastructure (see e.g. T. Brown et al., 2001; Garcia et al., 2008; B. Wang et al., 2002); (b) analysis of naturalistic driving data, for example to answer whatif? questions (see e.g. Davis \& Swenson, 2006; Fitch et al., 2008); (c) interpolation within, or extrapolation beyond, a limited data set of human driving (see e.g. Kuge et al., 2006; J. D. Lee et al., 2002); and (d) reconstruction of accidents (Araszewski et al., 2002; Sugimoto \& Sauer, 2005). 
Other authors have focused less on specific applications, but have instead used their driver models to formalize and test hypotheses on underlying psychological mechanisms (Salvucci \& Taatgen, 2008; Salvucci et al., 2004; possibly also e.g. Wada, Doi, et al., 2007; H. Yang \& Peng, 2010; Xin, Hourdos, Michalopoulos, $\&$ Davis, 2008). In other words, it seems that driver models may be of value in applications, both directly as components in simulation tools to be used by vehicle or infrastructure designers, and indirectly as components in research methodologies providing scientific knowledge that can shape guidelines for design.

Overall, it is clear that a wealth of different driver models has been proposed, with models of widely varying forms and modeling paradigms, from the simplest linear control laws to full cognitive architectures. For the researcher aiming to use or extend an existing driver model, the choice of one model from this seemingly fragmented field of research is not a trivial one, and no straightforward general recommendations can be provided here. It seems likely that the high complexity of driver behavior will continue to force researchers to limit their modeling scope so as to fit the specific application at hand, just as it has for the authors of the reviewed papers.

As an illustrative and important example, it may be noted that different authors have had to consider different application-specific requirements regarding which parts of the pre-crash timeline (Fig. 1) to cover. Many researchers have mainly been interested in the details of control in near-crash and crash phases, and have thus not needed to provide any account of why these states were reached in the first place (see e.g. Delaigue \& Eskandarian, 2004; Jurecki \& Stańczyk, 2009, and the large body of work on avoidance by steering). Other researchers have included in their scope also the low risk and conflict driving states, and have therefore needed to incorporate mechanisms causing transitions to the more critical states: Either visual distraction behavior (see the section on visual distraction, as well as e.g. Xin, Hourdos, Michalopoulos, \& Davis, 2008), or explicit error-generating mechanisms (see the section on simulation platforms, as well as H. Yang \& Peng, 2010). In relation to the qualitative models of accident causation presented in the background, it may be noted that the error-centric approach bears clear marks of the information processing paradigm (Wickens \& Hollands, 2000), whereas the visual distraction approach may be more closely tied to qualitative models such as that of Engström et al. (in press).

However, some models seem especially recommend- able for use in future work: (a) The delayed constant deceleration models are, despite (or thanks to) their simplicity, noteworthy for having proved useful in a wide range of applications. The inherent limitations with respect to less critical collision avoidance need to be taken into account, however. (b) If an application requires a braking model that can also exhibit non-critical, satisficing deceleration responses, the possible signs of convergence with empirical data seen in Figure 3 suggest that the models of Wada, Imai, et al. (2007); Wada, Doi, et al. (2007); Wada et al. (2009) or Gipps (1981) can provide good starting points. (c) The Xin, Hourdos, Michalopoulos, and Davis (2008) model, which builds on the Gipps model, is unique in that it is the only model in this review to have been fitted to time series data from actual crashes. (Davis \& Swenson, 2006 used the same data set, but placed less emphasis on driver modeling.) (d) Among the steering models, those that do not require explicit definition of a desired collision avoidance path seem preferable to us, assuming that they can be further validated on real crash-avoidance data (Gordon \& Best, 2006; Gordon \& Magnuski, 2006; Gao \& Jiang, 2009). (e) If a steering model using a desired path is preferred, the approach of Plöchl and Lugner (2000) to activate a specific mechanism in cases where path deviations become large is noteworthy, since a large instantaneous shift of the desired path is one possible conceptualization of what occurs in a collision emergency. (f) Given the current lack of validation of steering models on real accident situations, it cannot be excluded that simple open-loop responses, such as those proposed by Sugimoto and Sauer (2005) or Araszewski et al. (2002), are good enough for many applications. (g) The ACT$\mathrm{R}$ models of Salvucci (2006) and colleagues illustrate the potential benefits of adopting an existing cognitive architecture that has been subjected to much previous validation and tuning.

\section{Suggestions for future model development}

Comparing the reviewed models with the statements made in the background section of this paper, some suggestions can be made regarding possible areas for future model development work.

First of all, it may be noted that among the reviewed models, the models which address braking only are generally much simpler in their formulations than the models involving steering. For example, the braking models typically operate directly on vehicle acceleration or speed, rather than on the vehicle's pedals. For some applications, more detailed models of collision-avoidance braking control could therefore prove useful. 
Another observation that can be made is that although some authors have modeled reactions to collision warnings in various ways (e.g. Fitch et al., 2008; J. D. Lee et al., 2002; Steigerwald, 2002), none of the reviewed models have addressed the phenomena of $a c$ ceptance, reliance, and behavioral adaptation to longterm system exposure. This is not a trivial endeavor, but will be required if driver models are to be used for generating more than theoretical upper limits for predicted potential benefits of safety systems. Assuming a continued increase in proliferation of collision avoidance technology, these aspects of driver behavior are certainly worthy of modeling efforts.

Additionally, current technological trends point to an increased presence of support systems providing autonomous braking or steering interventions. Driver avoidance behavior in interaction with control interventions from the vehicle may take on qualitatively different forms than non-assisted avoidance, but this aspect has not been addressed in any of the reviewed papers (A possible exception is the paper by Kuge et al., 2006). Addressing this gap is desirable especially since intervening systems will require the type of rigorous testing for which simulation can be an important tool.

Furthermore, some of the reviewed models have been capable of behaviors such as visual distraction, and others have accounted for between-driver variability in collision avoidance control, but the issue of when and why drivers adopt risky behaviors, such as for example looking away from the road ahead, has not been addressed. In the terms used by Engström et al. (in press, presented in the background of this paper), this can be expressed as simulation models having focused mostly on the reactive barrier. In general, there is ample room for further research regarding simulations with driver models in the study of accident causation. Among the reviewed papers, only Davis and Swenson (2006) explicitly addressed such a goal, studying how rear-end crash responsibility may be attributable to more than one driver in a line of traffic. One possibility here could be to carry out simulations with driver models derived from competing qualitative models of accident causation, as a means of clarifying which qualitative models work best. Furthermore, several factors known to be involved in accident causation have received limited or no attention in the reviewed papers. For example, quantitative models of the effects of alcohol and fatigue on collision avoidance are absent altogether.

Another notable feature of many of the models, is the use of engineering practices not in line with current knowledge of human psychology. For example, rather than operating on the type of visual cues that human drivers seem to use (see e.g. Fajen, 2005; Wann $\&$ Wilkie, 2004), most reviewed models use high resolution data regarding, for example, longitudinal and lateral positions of vehicles. (Exceptions include the model by Wada, Doi, et al., 2007, and to some extent also those by H. Yang \& Peng, 2010, Xin, Hourdos, Michalopoulos, \& Davis, 2008, and Reński, 2001.) Furthermore, several driver models include features such as (a) preview, despite arguments that in urgent situations, control may shift to more short-sighted modes of operation (Hollnagel \& Woods, 2005), and (b) advanced internal vehicle models, despite observations that human drivers may not have a correct grasp of the dynamics of their vehicles (Cloete \& Wallis, 2009). In our opinion, psychology-oriented modelers could benefit from acknowledging that these types of practices can be powerful in the construction of phenomenological models, aimed at reproducing observed behavior data without necessarily making claims on underlying psychological (or neurobiological) mechanisms. It may however also be rightfully suggested to engineeringoriented modelers that models based on an understanding of such mechanisms could generalize better to wider scopes of application. In addition, it is our opinion that there may be logical pitfalls to avoid when using engineering methods, such as taking for granted that an inexperienced driver behaves as if having an internal vehicle model that is mathematically simple.

It may also be noted that the types of collision scenarios for which driver models have been developed remain limited in number, with a heavy focus on rearend scenarios. In order to achieve full credibility of simulation as a safety research approach, models will at some point need to address more diverse and complex pre-crash scenarios. Similarly, it would seem relevant to widen the modeling scope to also include collision-avoidance with other vehicles than passenger cars. (Among the reviewed papers, X. Yang et al., 2001 provided the only exception.) It could also be relevant to study to what extent models developed for one type of scenario or vehicle may be useful for other types.

Related to this issue, the mechanisms governing selection of maneuver type, as a function of the traffic situation, have not been given more than marginal attention among the reviewed models. The probabilities for braking versus steering in the model of Sugimoto and Sauer (2005) were not situation-dependent, and Jurecki and Stańczyk (2009) appear not to have put their statistical observations into simulation-ready model practice. Furthermore, although some of the reviewed mod- 
els could theoretically reproduce the no-maneuver response so often seen in real collision accidents (Wiacek \& Najm, 1999), this aspect has not been studied by any of the authors.

Thus, there may be a need for considering separate modes of collision avoidance control (Hollnagel \& Woods, 2005). Some of the reviewed models do include specific provisions for urgent situations (Plöchl \& Lugner, 2000; Yuhara \& Tajima, 2006; and possibly Akita et al., 2007), but an analysis of whether or not this is preferable is lacking. Currently it seems unclear whether critical avoidance is best modeled with (a) the same models as for normal avoidance, giving different response to the critical situation, (b) different parameterizations of the normal avoidance models, or (c) different models altogether.

In general, we would argue that none of the reviewed models have fully adopted the view of critical collisionavoidance maneuvering as a highly unexpected and unusual task. As suggested in the background above, behavior may become erratic or random in accident situations. Furthermore, if drivers do attempt more controlled maneuvers, their perceptual attunement to the critical situation may be limited, resulting in misinterpretations or maladjusted control actions. These aspects remain largely unexplored in current simulation models of driver behavior.

\section{Model validation and comparison}

Many of the reviewed models are capable of making highly detailed predictions on drivers' use of pedals and steering wheel during collision avoidance, as well as the effects on this control of various driver-related factors. However, validation of the models on relevant human data has rarely been achieved to the same level of detail. One important reason for this is probably that it is far from trivial to collect data on collision avoidance behavior, especially if the data are to be representative of behavior in unexpected collision situations in real traffic. Hopefully, recent and ongoing naturalistic driving studies (see e.g. Dingus et al., 2006) can provide researchers with better possibilities to achieve good model validation.

It is also clear, however, that even with good data, it is not evident how to carry out validation. In many papers, validation seems to have been limited to visual estimation of the match between time-series data on human and parameter-fitted driver model behavior. Although far beyond the scope of this discussion, more ambitious and rigorous methodologies for quantitative validation of driver models definitely seem to be needed. When the aim of a model is to test hypotheses on underlying psychological mechanisms, it needs to be shown that successful fits of observed data are not simply due to a highly flexible model (Roberts \& Pashler, 2000). In applied contexts, model flexibility may be less of a problem, but it still needs to be shown (e.g. by means of cross-validation or holdout validation techniques; see Hastie, Tibshirani, \& Friedman, 2009, p. 222) that a proposed parameter set is not the result of over-fitting to the human behavior data used. Another possible perspective is that, due to the stochastic nature of crashes, validation of models addressing near-collision control behavior may need to operate rather on distributions of trajectories or of other measures of behavior. For models covering also normal driving, the approach of comparing simulated crash frequency to accident statistics (see e.g. H. Yang \& Peng, 2010), may be one important part of a validation methodology.

Regardless of how the agreement between model and data is quantified, an important future development would be an increase in the practice of comparing driver models in actual simulation. Already the very basic comparisons presented in this review suggest dissimilarities and similarities which may not have been evident from the mathematical formulations of the compared models. The left panel of Figure 2 illustrates the fundamental difference between satisficing and nonsatisficing models of braking. Figure 3 indicates a possible convergence, in terms of non-critical brake initiation timing, between the models of Gipps (1981) and Wada et al. (2009) on the one hand, and the data set of Kiefer et al. (2005) on the other. For researchers who are mainly interested in whether or not a given simulated scenario results in a collision or not, Figure 4 can be taken to suggest that it may be enough to adopt a rather simple model of avoidance by steering: For example, all models would have avoided a stationary obstacle at a longitudinal position of $40 \mathrm{~m}$.

In our opinion, comparison of models ought to be much more frequent in this research field than it currently is. As is clear from the present review, for a given traffic scenario or behavioral phenomenon, there are often several competing driver models, but it is rarely known how these models differ in terms of their behavior, or in terms of how well they are able to reproduce the corresponding behavior of human drivers. In order for novel near-collision behavior models to be of value, either from a scientific or an applied point of view, their development should be complemented with comparative investigations. Some model developers who have set good examples in this respect are K. Lee and 
Peng (2004), Ungoren and Peng (2005), and MacAdam (2003).

\section{Concluding remarks}

We have provided a review of recent simulationready models of driver behavior in accident situations involving on-road collisions. The results show a somewhat fragmented research field, in which many different models have been proposed for a wide variety of applications. However, based on the results obtained from simulations of existing models, we suggest that, in some cases, there may be more similarity between the models than what is immediately apparent from the corresponding equations.

Some models have been identified that may deserve attention in future work. However, it has also been emphasized that, due to the complexity of the processes being modeled, it seems likely that for the foreseeable future, the scope of requirements to set for a driver model will need to be strictly limited to fit the intended context of application.

Specific suggestions for future work on model development have been made, but it has also been argued that a major remaining challenge is an improved paradigm for validation and comparison of already existing models.

\section{Key points}

- Computer simulation of accident situations holds promise as a valuable tool for traffic safety research.

- This paper is a review of near-collision driver behavior models that are suitable for use in computer simulation.

- A wide variety of models has been proposed, but validation on collision-relevant human behavior data has so far often been limited.

- Simulation-based comparison suggests some nontrivial similarities between existing models, and further comparison of this kind is recommended.

\section{References}

Adams, L. D. (1994). Review of the literature on obstacle avoidance maneuvers: braking versus steering (Tech. Rep. No. UMTRI-94-19). The University of Michigan Transportation Research Institute.

Akita, T., Inagaki, S., Suzuki, T., Hayakawa, S., \& Tsuchida, N. (2007, Oct). Analysis of vehicle following behavior of human driver based on hybrid dynamical system model. In Proceedings of the 16th IEEE international conference on control applications (pp. 1233-1238).
Allen, R. W. (1982). Stability and performance analysis of automobile driver steering control (Tech. Rep. No. 820303). SAE.

Anderson, J. R., \& Lebiere, C. (1998). The atomic components of thought. Mahwah, NJ: Erlbaum.

Araszewski, M., Toor, A., Overgaard, R., \& Johal, R. (2002). Lane change maneuver modelling for accident reconstruction applications (Tech. Rep. No. 2002-01-0817). SAE International.

Benderius, O., Markkula, G., Wolff, K., \& Wahde, M. (2011). A simulation environment for analysis and optimization of driver models. In Proceedings of the 14th international conference on human-computer interaction (pp. 453-462).

Brackstone, M., \& McDonald, M. (1999). Car-following: a historical review. Transportation Research Part F: Traffic Psychology and Behaviour, 2(4), 181-196.

Breuer, J. (1998). Analysis of driver-vehicle-interactions in an evasive manoeuvre - results of 'moose test' studies. In Proceedings of the 15th ESV conference (pp. 620-627).

Brown, T., Lee, J., \& McGehee, D. (2000). Attentionbased model of driver performance in rear-end collisions. Transportation Research Record: Journal of the Transportation Research Board, 1724, 14-20.

Brown, T., Lee, J., \& McGehee, D. (2001). Human performance models and rear-end collision avoidance algorithms. Human Factors: The Journal of the Human Factors and Ergonomics Society, 43(3), 462-482.

Brown, T. L., Lee, J. D., \& McGehee, D. V. (2001). Validation of an attention-based model of driver performance in rear-end collision situations. In Proceedings of the TRB 80th annual meeting, Washington DC, United States.

Butz, T., \& von Stryk, O. (2005). Optimal control based modeling of vehicle driver properties (Tech. Rep. No. 2005-01-0420). SAE International.

Chatzikomis, C., \& Spentzas, K. (2009). A path-following driver model with longitudinal and lateral control of vehicle's motion. Forschung im Ingenieurwesen, 73(4), 257266.

Chung, S., Song, K., Hong, S., \& Kho, S. (2005). Development of sensitivity term in car-following model considering practical driving behavior of preventing rear end collision. Journal of the Eastern Asia Society for Transportation Studies, 6, 1354-1367.

Cloete, S., \& Wallis, G. (2009). Limitations of feedforward control in multiple-phase steering movements. Experimental Brain Research, 195(3), 481-487.

Cody, D., \& Gordon, T. (2007). TRB workshop on driver models: A step towards a comprehensive model of driving? In P. Cacciabue (Ed.), Modelling driver behaviour in automotive environments: Critical issues in driver interactions with intelligent transport systems. Springer.

Cooper, P. J., \& Zheng, Y. (2002). Turning gap acceptance decision-making: the impact of driver distraction. Journal of Safety Research, 33, 321-335.

Data, S., Pascali, L., \& Santi, C. (2002). Handling objective evaluation using a parametric driver model for ISO lane 
change simulation (Tech. Rep. No. 2002-01-1569). SAE International.

Davis, G. (2007). On the plausibility of using simulation to model left-turn cross-path crashes. In TRB 86th annual meeting compendium of papers.

Davis, G., \& Swenson, T. (2006). Identification and simulation of a common freeway accident mechanism: Collective responsibility in freeway rear-end collisions (Final Report No. CTS 06-02). Center for Transportation Studies, University of Minnesota.

Delaigue, P., \& Eskandarian, A. (2004). A comprehensive vehicle braking model for predictions of stopping distances. Proceedings of the Institution of Mechanical Engineers, Part D: Journal of Automobile Engineering, 218(12), 1409-1417.

Delorme, D., \& Song, B. (2001). Human driver model for smartAHS (California PATH Research Report No. UCBITS-PRR-2001-12). University of California, Berkeley, U.S.

Dilich, M., Kopernik, D., \& Goebelbecker, J. (2002). Evaluating driver response to a sudden emergency: Issues of expectancy, emotional arousal and uncertainty (Tech. Rep. No. 2002-01-0089). SAE International.

Dingus, T., Klauer, S., Neale, V., Petersen, A., Lee, S., Sudweeks, J., ... Knipling, R. (2006). The 100-car naturalistic driving study, phase II - results of the 100-car field experiment (Interim Report No. DOT HS 810 593). Virginia Tech Transportation Institute.

Donges, E. (1978). A two-level model of driver steering behavior. Human Factors: The Journal of the Human Factors and Ergonomics Society, 20(6), 691-707.

Edelmann, J., Plöchl, M., Reinalter, W., \& Tieber, W. (2007). A passenger car driver model for higher lateral accelerations. Vehicle System Dynamics, 45(12), 1117-1129.

El Hajjaji, A., \& Ouladsine, M. (2001). Modeling human vehicle driving by fuzzy logic for standardized ISO double lane change maneuver. In Proceedings of the 10th IEEE international workshop on robot and human interactive communication (pp. 499-503).

Engström, J. (2011). Understanding attention selection in driving: From limited capacity to adaptive behavior. $\mathrm{PhD}$ thesis, Chalmers University of Technology.

Engström, J., Victor, T. W., \& Markkula, G. (in press). Attention selection and multitasking in everyday driving: A conceptual model. In M. A. Regan, T. W. Victor, \& J. D. Lee (Eds.), Driver distraction and inattention: Advances in research and countermeasures. Ashgate.

Erséus, A. (2010). Driver-vehicle interaction : Identification, characterization and modelling of path tracking skill. $\mathrm{PhD}$ thesis, KTH.

Fajen, B. R. (2005). Calibration, information, and control strategies for braking to avoid a collision. Journal of Experimental Psychology: Human Perception and Performance, 31(3), 480-501.

Fajen, B. R. (2008). Perceptual learning and the visual control of braking. Perception \& Psychophysics, 70(6), 1117-1129.
Fambro, D., Fitzpatrick, K., \& Koppa, R. (2000). New stopping sight distance model for use in highway geometric design. Transportation Research Record: Journal of the Transportation Research Board, 1701, 1-8.

Fitch, G. M., Rakha, H. A., Arafeh, M., Blanco, M., Gupta, S. K., Zimmermann, R., \& Hanowski, R. J. (2008). Safety benefit evaluation of a forward collision warning system (Final Report No. DOT HS 810 910). U.S. Department of Transportation.

Furukawa, Y., Seki, K., \& Fujikawa, T. (2009). Development of traffic simulator using universal driver model for safety evaluation of ADAS. In Proceedings of the 16th world congress on ITS, Stockholm, Sweden.

Gao, Z., \& Jiang, L. (2009). Optimal preview trajectory decision model of lane-keeping system with driver behavior simulation and artificial potential field. In Intelligent vehicles symposium, 2009 ieee (pp. 797-801).

Gao, Z., Zheng, N., Guan, H., \& Guo, K. (2002). Application of driver direction control model in intelligent vehicle's decision and control algorithm. Intelligent Vehicle Symposium, 2002. IEEE, 2, 413-418.

Garcia, A., Libreros, L., \& Contreras, J. (2008). A new microsimulator to evaluate road safety at skewed intersections. Advances in Transportation Studies: an International Journal, Section B, 14, 81-88.

Gazis, D., Herman, R., \& Rothery, R. (1961). Nonlinear follow-the-leader models of traffic flow. Operations Research, 9(4), 545-567.

Ghazi Zadeh, A., Fahim, A., \& El-Gindy, M. (1997). Neural network and fuzzy logic applications to vehicle systems: literature survey. International Journal of Vehicle Design, $18(2), 132-93$.

Gibson, J., \& Crooks, L. (1938). A theoretical field-analysis of automobile-driving. The American Journal of Psychology, 51(3), 453-471.

Gipps, P. (1981). A behavioural car-following model for computer simulation. Transportation Research Part B: Methodological, 15B, 105-111.

Gordon, T., \& Best, M. (2006). On the synthesis of driver inputs for the simulation of closed-loop handling manoeuvres. International Journal of Vehicle Design, 40(1-3), 52-76.

Gordon, T., \& Magnuski, N. (2006). Modeling normal driving as a collision avoidance process. In Proceedings of 8 th international symposium on advanced vehicle control.

Gray, P. (2010). Psychology (6th ed.). Worth Publishers.

Green, M. (2000). "How long does it take to stop?" Methodological analysis of driver perception-brake times. Transportation Human Factors, 2(3), 195-216.

Guo, K., Ding, H., Zhang, J., Lu, J., \& Wang, R. (2004). Development of a longitudinal and lateral driver model for autonomous vehicle control. International Journal of Vehicle Design, 36, 50-65.

Guo, K., \& Fancher, P. (1983). Preview-follower method for modeling closed-loop vehicle directional control. In Proceedings of the 19th annual conference on manual control, cambridge, massachusetts (pp. 158-187). 
Hamdar, S., \& Mahmassani, H. (2005). Modeling driver behavior under extreme conditions. In Proceedings of the 12th World Congress on ITS, November 2005, San Francisco, California, United States.

Hastie, T., Tibshirani, R., \& Friedman, J. (2009). The elements of statistical learning (Second ed.). Springer.

Helbing, D. (2001). Traffic and related self-driven manyparticle systems. Reviews of modern physics, 73(4), 1067-1141.

Hollnagel, E., \& Woods, D. D. (2005). Joint cognitive systems: Foundations of cognitive systems engineering. CRC Press / Taylor \& Francis.

Irmscher, M., \& Ehmann, M. (2004). Driver classification using ve-DYNA advanced driver (Tech. Rep. No. 200401-0451). SAE International.

ISO. (1999). Passenger cars - test track for a severe lanechange manoeuvre - part 1: Double lane-change (No. 3888).

Itoh, M., Horikome, T., \& Inagaki, T. (2010). Effectiveness and driver acceptance of a semi-autonomous forward obstacle collision avoidance system. In Proceedings of the human factors and ergonomics society 54th annual meeting.

Jürgensohn, T. (2007). Control theory models of the driver. In P. Cacciabue (Ed.), Modelling driver behaviour in automotive environments (pp. 277-292). Springer.

Jurecki, R., \& Stańczyk, T. (2009). Driver model for the analysis of pre-accident situations. Vehicle System Dynamics, 47(5), 589-612.

Kiefer, R., LeBlanc, D., \& Flannagan, C. (2005). Developing an inverse time-to-collision crash alert timing approach based on drivers' last-second braking and steering judgments. Accident Analysis \& Prevention, 37(2), 295-303.

Kim, J., Hayakawa, S., Suzuki, T., Hayashi, K., Okuma, S., Tsuchida, N., ... Kido, S. (2005). Modeling of driver's collision avoidance maneuver based on controller switching model. IEEE Transactions on Systems, Man, and Cybernetics, Part B: Cybernetics, 35(6), 1131-1143.

Kitaoka, H., Kurahashi, T., Mori, H., Iwase, T., Machida, T., Kozato, A., ... Yoshikatsu, K. (2009). A development of a traffic simulator for safety evaluation - reproduction of traffic accidents and evaluation of safety systems. Review of Automotive Engineering, 30(2), 211-217.

Kleinman, D. L., Baron, S., \& Levison, W. H. (1970). An optimal control model of human response part i: Theory and validation. Automatica, 6(3), 357-369.

Krishnan, H., Gibb, S., Steinfeld, A., \& Shladover, S. (2001). Rear-end collision-warning system: Design and evaluation via simulation. Transportation Research Record: Journal of the Transportation Research Board, 1759, 5260.

Kuge, N., Yamamura, T., Boer, E. R., Ward, N. J., \& Manser, M. P. (2006). Study on driver's car following abilities based on an active haptic support function (Tech. Rep. No. 2006-01-0344). SAE International.

Land, M., \& Horwood, J. (1995). Which parts of the road guide steering? Nature, 377, 339-340.
Lechner, D., \& van Elslande, P. (1997). Comportement du conducteur en situation d'accident (Tech. Rep. No. 199719-0084). SAE International.

Lee, D. (1976). A theory of visual control of braking based on information about time-to-collision. Perception, 5(4), 437-459.

Lee, J. D. (2008). Fifty years of driving safety research. Human Factors: The Journal of the Human Factors and Ergonomics Society, 50(3), 521-528.

Lee, J. D., McGehee, D. V., Brown, T. L., \& Reyes, M. L. (2002). Collision warning timing, driver distraction, and driver response to imminent rear-end collisions in a highfidelity driving simulator. Human Factors: The Journal of the Human Factors and Ergonomics Society, 44(2), 314335.

Lee, J. D., \& See, K. A. (2004). Trust in automation: Designing for appropriate reliance. Human Factors: The Journal of the Human Factors and Ergonomics Society, 46(1), 50-80.

Lee, K., \& Peng, H. (2004). Identification and verification of a longitudinal human driving model for collision warning and avoidance systems. International Journal of Vehicle Autonomous Systems, 2(1), 3-17.

Lee, S. E., Llaneras, E., Klauer, S., \& Sudweeks, J. (2007, Oct). Analyses of rear-end crashes and near-crashes in the 100-car naturalistic driving study to support rearsignaling countermeasure development (Tech. Rep. No. DOT HS 810 846). Virginia Tech Transportation Institute.

Lin, M., Popov, A., \& McWilliam, S. (2003). Sensitivity analysis of driver characteristics in driver-vehicle handling studies. In L. Dorn (Ed.), Driver behaviour and training (pp. 263-276). Ashgate Publishing Ltd.

Lin, Y., Tang, P., Zhang, W., \& Yu, Q. (2005). Artificial neural network modelling of driver handling behaviour in a driver-vehicle-environment system. International Journal of Vehicle Design, 37(1), 24-45.

MacAdam, C. (1981). Application of an optimal preview control for simulation of closed-loop automobile driving. IEEE Transactions on Systems, Man, and Cybernetics, 11(6), 393-399.

MacAdam, C. (2001). Development of a driver model for nearlat-limit vehicle handling (Final report No. UMTRI2001-43). University of Michigan Transportation Research Institute.

MacAdam, C. (2003, Jan). Understanding and modeling the human driver. Vehicle System Dynamics, 40(1-3), 101134.

Malaterre, G., Ferrandez, F., Fleury, D., \& Lechner, D. (1988). Decision making in emergency situations. Ergonomics, 31(4), 643-655.

McDonald, M., Wu, J., \& Brackstone, M. (1997). Development of a fuzzy logic based microscopic motorway simulation model. In Proceedings of the 1997 IEEE conference on intelligent transportation system (pp. 82-87).

McGehee, D. V., Mazzae, E. N., Baldwin, G. H. S., Grant, P., Simmons, C. J., Hankey, J., \& Forkenbrock, G. (1999). Examination of Drivers' Collision Avoidance Behavior 
Using Conventional and Antilock Brake Systems on the Iowa Driving Simulator (Final Report). University of Iowa.

McMillan, N. J., Christiaen, A.-C., \& Stark, G. V. (2001). Estimating safety benefits for the IVI generation 0 field operational tests (Tech. Rep. No. 2001-01-2818). SAE International.

Mehmood, A., Saccomanno, F., \& Hellinga, B. (2001). Simulation of road crashes by use of systems dynamics. Transportation Research Record: Journal of the Transportation Research Board, 1746, 37-46.

Meyer, J. (2004). Conceptual issues in the study of dynamic hazard warnings. Human Factors: The Journal of the Human Factors and Ergonomics Society, 46(2), 196-204.

Michon, J. (1985). A critical view of driver behavior models: What do we know, what should we do? In L. Evans \& R. Schwing (Eds.), Human behavior and traffic safety (pp. 485-520). Plenum, New York.

Muttart, J. (2003). Development and evaluation of driver response time predictors based upon meta analysis (Tech. Rep. No. 2003-01-0885). SAE International.

Najm, W., \& Smith, D. (2004). Modelling driver response to lead vehicle decelerating (Tech. Rep. No. 2004-01-0171). SAE International.

Najm, W., Smith, J., \& Yanagisawa, M. (2007). Pre-crash scenario typology for crash avoidance research (Final report No. DOT HS 810 767). U.S. Department of Transportation.

Noh, K., Jung, D., Choi, H., \& Yi, K. (2007). Development of ergonomic driver model considering human factors (Tech. Rep. No. 2007-01-3584). SAE International.

Oppenheim, I., Shinar, D., Carsten, O., Barnard, Y., Lai, F., Vanderhaegen, F., ... Kecklund, L. (2010). Critical review of models and parameters for driver models in different surface transport systems and in different safety critical situations (Deliverable No. D1.1). EU FP7 ITERATE Project. Retrieved 2011-06-08, from http: / / www. iterate-project.eu/

Ozaki, H. (1993). Reaction and anticipation in the car following behaviour. In Proceedings of the 13th international symposium on traffic and transportation theory (pp. 349-366).

Peng, H. (2002). Evaluation of driver assistance systems - a human centered approach. In AVEC proceedings.

Plöchl, M., \& Edelmann, J. (2007). Driver models in automobile dynamics application. Vehicle System Dynamics, 45(7-8), 699-741.

Plöchl, M., \& Lugner, P. (2000). 3-level driver model and its application to driving simulations. Vehicle System Dynamics, 33, 71-82.

Prokop, G. (2001). Modeling human vehicle driving by model predictive online optimization. Vehicle System Dynamics, 35(1), 19-53.

Ranney, T. (1994). Models of driving behavior: a review of their evolution. Accident Analysis \& Prevention, 26(6), $733-750$

Reid, L. (1983). A survey of recent driver steering behavior models suited to accident studies. Accident Analysis \&
Prevention, 15(1), 23-40.

Reński, A. (2001). Identification of driver model parameters. International Journal of Occupational Safety and Ergonomics, 7(1), 79-90.

Roberts, S., \& Pashler, H. (2000). How persuasive is a good fit? a comment on theory testing. Psychological Review, 107(2), 358-367.

Saka, A., \& Glassco, R. (2001). Modeling traffic safety benefits of intelligent transportation system technologies at truck inspection facilities: Microscopic simulation. Transportation Research Record: Journal of the Transportation Research Board, 1779, 173-181.

Salvucci, D. (2002). Modeling driver distraction from cognitive tasks. In Proceedings of the 24th annual conference of the cognitive science society (pp. 792-797).

Salvucci, D. (2006). Modeling driver behavior in a cognitive architecture. Human Factors: The Journal of the Human Factors and Ergonomics Society, 48(2), 362-380.

Salvucci, D., Chavez, A., \& Lee, F. (2004). Modeling effects of age in complex tasks: A case study in driving. In Proceedings of the 26th annual conference of the cognitive science society (pp. 1197-1202).

Salvucci, D., \& Taatgen, N. (2008). Threaded cognition: An integrated theory of concurrent multitasking. Psychological Review, 115, 101-130.

Sharp, R., Casanova, D., \& Symonds, P. (2000). A mathematical model for driver steering control, with design, tuning and performance results. Vehicle System Dynamics, 33(5), 289-326.

Smiley, A. (2000). Behavioral adaptation, safety, and intelligent transportation systems. Transportation Research Record: Journal of the Transportation Research Board, 1724, 47-51.

Smith, D., Chang, J., Cohen, D., Foley, J., \& Glassco, R. (2005). A simulation approach for evaluating the relative safety impact of driver distraction during secondary tasks. In Proceedings of the 12th world congress on ITS, 6-10 November 2005, San Francisco, United States.

Steigerwald, D. (2002). RECmodeler - evaluating cooperative collision avoidance. In Proceedings of ITS America 12th annual meeting and exposition, Long Beach, California, United States.

Sugimoto, Y., \& Sauer, C. (2005). Effectiveness estimation method for advanced driver assistance system and its application to collision mitigation brake system. In Proceedings of the 19th international technical conference on the enhanced safety of vehicles, Washington DC, United States.

Sultan, B., Brackstone, M., \& McDonald, M. (2004). Drivers' use of deceleration and acceleration information in car-following process. Transportation Research Record: Journal of the Transportation Research Board, 1883, 31-39.

Summala, H. (2007). Towards understanding motivational and emotional factors in driver behaviour: Comfort through satisficing. In P. Cacciabue (Ed.), Modelling driver behaviour in automotive environments (pp. 189207). Springer. 
Thommyppillai, M., Evangelou, S., \& Sharp, R. (2009). Car driving at the limit by adaptive linear optimal preview control. Vehicle System Dynamics, 47(12), 1535-1550.

Ungoren, A., \& Peng, H. (2005). An adaptive lateral preview driver model. Vehicle System Dynamics, 43(4), 245-259.

van Elslande, P., \& Fouquet, K. (2007). Analyzing 'human functional failures' in road accidents (Deliverable No. 5.1). TRACE EU Project. Retrieved 2011-06-07, from http: / / www.trace-project.org/

Vögel, M., von Stryk, O., Bulirsch, R., Wolter, T., \& Chucholowski, C. (2003). An optimal control approach to real-time vehicle guidance. In W. Jäger \& H. Krebs (Eds.), Mathematics - key technology for the future (pp. 84-102). Springer Berlin Heidelberg.

Vinter, R. (2010). Optimal control. Birkhäuser.

Wada, T., Doi, S., Imai, K., Tsuru, N., Isaji, K., \& Kaneko, H. (2007). On driver's braking behavior in car following. In Proceedings of SICE annual conference (pp. 2396-2401). Takamatsu.

Wada, T., Hiraoka, S., \& Doi, S. (2009). A deceleration assistance control for collision avoidance based on driver's perceptual risk. In Proceedings of the 2nd international conference on intelligent robotics and applications (Vol. 5928 LNAI, pp. 215-225). Singapore.

Wada, T., Imai, K., Tsuru, N., Isaji, K., \& Kaneko, H. (2007). Analysis of drivers' behaviors in car following based on a performance index for approach and alienation (Tech. Rep. No. 2007-01-0440). SAE International.

Wang, B., Abe, M., \& Kano, Y. (2002). Influence of driver's reaction time and gain on driver-vehicle system performance with rear wheel steering control systems: part of a study on vehicle control suitable for the aged driver. JSAE Review, 23, 75-82.

Wang, X., Yang, X., Shan, G., \& Wang, F. (2006). Review of the simulation model of driving behavior. In Proceedings of the international conference on machine learning and cybernetics (pp. 911-918).

Wann, J. P., \& Wilkie, R. M. (2004). How do we control high speed steering? In L. M. Vaina, S. A. Beardsley, \& S. K. Rushton (Eds.), Optic flow and beyond (pp. 371389). Dordrecht: Kluwer Academic Publishers.

Weir, D. H., \& Chao, K. C. (2007). Review of control theory models for directional and speed control. In P. Cacciabue (Ed.), Modelling driver behaviour in automotive environments: Critical issues in driver interactions with intelligent transport systems. Springer.

Wenzel, T., Burnham, K., Williams, R., \& Blundell, M. (2005). Closed-loop driver/vehicle model for automotive control. In Proceedings of the 18th international conference on systems engineering (pp. 46-51).

Wiacek, C., \& Najm, W. (1999). Driver/vehicle characteristics in rear-end precrash scenarios based on the general estimates system (Tech. Rep. No. 1999-01-0817). SAE International.

Wickens, C. D., \& Hollands, J. G. (2000). Engineering psychology and human performance (3rd ed.). PrenticeHall.
Wood, R., Dumbuya, A., Zhao, J., Hill, J., \& Thomas, P. (2003). Simulation of driver, vehicle and environmental aspects of crash initiation, a new method to improve integrated safety effectiveness. In Proceedings of the 18th international technical conference on the enhanced safety of vehicles.

World Health Organization. (2009). Global status report on road safety: Time for action. Retrieved 201106-08, from www.who.int/violence_injury _prevention/road_safety_status/2009

Xin, W., Hourdos, J., \& Michalopoulos, P. (2008). Enhanced micro-simulation models for accurate safety assessment of traffic management ITS solutions (Final report No. CTS 08-17). Center for Transportation Studies, University of Minnesota.

Xin, W., Hourdos, J., Michalopoulos, P., \& Davis, G. (2008). The less-than-perfect driver: a model of collisioninclusive car-following behavior. Transportation Research Record: Journal of the Transportation Research Board, 2088, 126-137.

Yamakado, M., \& Abe, M. (2008). An experimentally confirmed driver longitudinal acceleration control model combined with vehicle lateral motion. Vehicle System Dynamics, 46(S1), 129-149.

Yan, X., Harb, R., \& Radwan, E. (2008). Analyses of factors of crash avoidance maneuvers using the general estimates system. Traffic Injury Prevention, 9(2), 173-180.

Yang, H., \& Peng, H. (2010). Development of an errorable car-following driver model. Vehicle System Dynamics, 48(6), 751-773.

Yang, X., Rakheja, S., \& Stiharu, I. (2001). Adapting an articulated vehicle to its drivers. Journal of Mechanical Design, 123(1), 132-140.

Yoshida, H., Shinohara, S., \& Nagai, M. (2008). Lane change steering manoeuvre using model predictive control theory. Vehicle System Dynamics, 46(S1), 669-681.

Yuhara, N., \& Tajima, J. (2006). Multi-driver agent-based traffic simulation systems for evaluating the effects of advanced driver assistance systems on road traffic accidents. Cognition, Technology \& Work, 8(4), 283-300.

Zadeh, L. A. (1965). Fuzzy sets. Inf. and Control, 8, 338353.

Zeyada, Y., El-Beheiry, E., El-Arabi, M., \& Karnopp, D. (2000). Driver modeling using fuzzy logic controls for human-in-the-loop vehicle simulations. In Current advances in mechanical design and production VII: proceedings of the seventh Cairo University international MDP conference (pp. 85-94).

Zhuang, D., Yu, F., \& Li, D. (2005). An optimal preview ANN driver model based on error elimination algorithm (Tech. Rep. No. 2005-01-3495). SAE International.

Gustav Markkula is currently with the Vehicle Solutions and Transport Analysis group at Volvo Technology Corporation. In 2004, he obtained a MSc in Engineering Physics from Chalmers University of Technology, where he is now pursuing a $\mathrm{PhD}$ in Machine and 
Vehicle Systems.

Ola Benderius is currently with the Department of Applied Mechanics at Chalmers University of Technology. He obtained a MSc in Computer Science from Chalmers University of Technology in 2009, and is now pursuing a PhD in Machine and Vehicle Systems.

Dr. Krister Wolff is currently with the Department of Applied Mechanics at Chalmers University of Technology. He obtained a PhD in Machine and Vehicle Systems from Chalmers University of Technology in 2006.

Prof. Mattias Wahde is currently with the Department of Applied Mechanics at Chalmers University of Technology. He obtained a PhD in Mechanics from Chalmers University of Technology in 1997.

\section{Appendix A}

\section{Literature database searches}

Databases searched: ARL, IEEE Xplore, ISI Web of Science, Inspec, PubMed, SAE, Scopus, TRIS.

Search queries varied depending on search syntax and the features of the individual databases. Example for Scopus: TITLE-ABS-KEY ( collision* or accident* or incident* or safety or "driv* support" or "driv* assistance") AND (simulat* or

quantitative or mathematic* or

model*) AND (driver W/6 model*)) AND

PUBYEAR AFT 1999

Appendix B

Vehicle model parameters

\section{Table B1}

: Vehicle model parameters (Thommyppillai et al., 2009, p. 1538), used when simulating driver steering models (see Figure 4).

\begin{tabular}{lcrc}
\hline Parameter & Symbol & Value & Unit \\
\hline Mass & $M$ & 1400 & $\mathrm{~kg}$ \\
Yaw inertia & $I_{z}$ & 2500 & $\mathrm{~kg} \mathrm{~m}^{2}$ \\
Front axle distance & $a$ & 1.16 & $\mathrm{~m}$ \\
Rear axle distance & $b$ & 1.54 & $\mathrm{~m}$ \\
Steering gear ratio & $G$ & 17 & - \\
Stiffness factor & $B_{m}$ & 11.5 & - \\
Shape factor & $C_{m}$ & 1.3 & - \\
Peak factor & $D_{m}$ & 2500 & $\mathrm{~N}$ \\
Curvature factor & $E_{m}$ & 0.3 & -
\end{tabular}

